\title{
Relativistic quantum effects of Dirac particles simulated by ultracold atoms
}

\author{
Dan-Wei Zhang ${ }^{1,2}$, Z. D. Wang ${ }^{2}$, Shi-Liang Zhu ${ }^{1} \cdot$. \\ ${ }^{1}$ Laboratory of Quantum Information Technology, \\ School of Physics and Telecommunication and Engineering, \\ South China Normal University, Guangzhou, China \\ ${ }^{2}$ Department of Physics and Center of Theoretical and Computational Physics, \\ The University of Hong Kong, Pokfulam Road, Hong Kong, China
}

(Dated: July 23, 2018)

\begin{abstract}
Quantum simulation is a powerful tool to study a variety of problems in physics, ranging from high-energy physics to condensed-matter physics. In this article, we review the recent theoretical and experimental progress in quantum simulation of Dirac equation with tunable parameters by using ultracold neutral atoms trapped in optical lattices or subject to light-induced synthetic gauge fields. The effective theories for the quasiparticles become relativistic under certain conditions in these systems, making them ideal platforms for studying the exotic relativistic effects. We focus on the realization of one, two, and three dimensional Dirac equations as well as the detection of some relativistic effects, including particularly the well-known Zitterbewegung effect and Klein tunneling. The realization of quantum anomalous Hall effects is also briefly discussed.
\end{abstract}

Keywords: ultracold atoms, Dirac equation, quantum simulation

PACS numbers: 67.85.-d, 31.15.6t, 47.27.ek

Contents

\section{Introduction}

II. Simulation of Dirac equation with ultracold atoms in OL systems

A. Simulation of 2D relativistic Dirac fermions

1. Mimic graphene: Cold atoms in a honeycomb OL

2. Pseudospin-1 massless Dirac fermions: Atoms in a $\mathcal{T}_{3} /$ line-centered-square $\mathrm{OL}$

3. Dirac fermions in a square OL with a gauge field

B. Simulation of 3D relativistic Dirac fermions

C. Detection of the Dirac quasiparticles

D. Quantum anomalous Hall effects

III. Simulation of Dirac equation with ultracold atoms in synthetic non-Abelian gauge fileds 10

A. Synthetic gauge fields for neutral atoms

B. Tunable Dirac-type equation for cold atoms with tripod- and $\Lambda$ - level structure

C. Simulation of nonlinear Dirac equation

IV. Observation of some relativistic effects
A. Zitterbewegung
B. Klein tunneling
C. Macroscopic Klein tunneling

V. Conclusions and perspectives

References

*Electronic address: slzhu@scnu.edu.cn

\section{INTRODUCTION}

As first pinpointed by Richard Feynman in 1982, a quantum computer could be used to simulate a quantum system efficiently without experiencing an exponential explosion and slowdown, which a classic computer would presumably encounter when simulating quantum phenomena [1]. Although quantum computers are not yet available, one can still hope to create analog quantum simulators which are designed to have the same Hamiltonian as another system of interest. The recent rapid progress in quantum coherent control [2] of neutral atoms, photons, or ions in some systems makes them become ideal quantum simulators [3], which include neutral atoms in optical lattices (OLs), arrays of cavities, trapped ions, quantum dots, superconducting circuits, nuclear magnetic resonance (NMR) and so on.

Analog quantum simulators would be able to provide highly controllable platforms to study difficult problems in various fields, ranging from high-energy physics, condensed-matter physics to quantum chemistry. Some recent notable achievements in quantum simulation are listed as follows: addressing neutral atoms in OLs to simulate the Bose-Hubbard model with the quantum phase transition from a superfluid to a Mott insulator [4-6], as well as to simulate the antiferromagnetic spin chains [7]; simulating a quantum magnet [8], frustrated Ising model [9], relativistic dynamics [10,11], and opensystem dynamics [12] with trapped ions; implementing a hydrogen molecule quantum simulation with NMR [13]. Besides, many other experimental proposals of quantum simulation with different simulators are suggested (see the review [3] and the references therein).

One of the most promising quantum simulators is the cold atom system since the realization of condensation 
of bosonic atoms $[14,15]$ and fermionic atom pairs $[16,17]$ has led to numerous advances with applications in coherent manipulation of neutral atoms, such as creating cold molecules in quantum chemistry (see the reviews $[18,19]$ and the references therein). Ultracold neutral atoms in OLs are quite suited for mimicking condensed-matter physics [20,21], and may shew new light on strongly correlated state problems like high-temperature superconductivity [22], fractional quantum Hall effect [23,24], and the quantum magnetism [25]. Ultracold atomic gases in disordered optical potentials pave the way towards the realization of versatile quantum simulators for the investigations of Anderson localization [26,27], which may help solve some open questions relying on the interplay of disorder and interactions [28,29]. In addition, some phenomena and effects in high-energy physics and cosmology can be mimicked, such as quantum simulation of black holes [30] and cosmic inflation [31] by using BoseEinstein condensates (BECs), or superstrings [32] and supersymmetry $[33,34]$ by using Bose-Fermi mixtures.

Since the relativistic Dirac fermions were found in graphene $[35,36]$, a substantial amount of efforts has been devoted to the understanding of exotic relativistic effects in solid state systems and the searching for other relativistic systems such as the recent discovery of topological insulators [37]. Inspired by those exciting results and the state-of-the-art technologies in quantum control of atoms, one specific topic among ultracold neutral atom simulators has arisen, that is, quantum simulation of the Dirac equation and the related relativistic effects.

The Dirac equation $i \hbar \partial_{t} \Psi=\left(c \vec{\alpha} \cdot \overrightarrow{\mathbf{p}}+m c^{2} \beta\right) \Psi$, where $\vec{\alpha}$ and $\vec{\beta}$ are the Dirac matrices, and $c$ is the speed of light, was first proposed by Dirac who was seeking a relativistic and quantum mechanical equation to describe spin $1 / 2$ particles with mass $m$. This equation naturally provides a description of the electron spin, which is an assumption in the Schrödinger equation. More surprisingly, it predicts the existence of antiparticles [38], which was soon confirmed by the experimental discovery of positrons. The Dirac equation also predicts some exotic effects, and the most famous ones are Zitterbewegung (ZB) [39], an unexpected trembling motion of free relativistic particles, and Klein tunneling (KT) [40], which describes relativistic particles penetrating through high and wide potential barriers without exponential damping expected in nonrelativistic tunneling processes. Despite these relativistic effects have attracted lots of interest over years, they are failed to be directly tested by elementary particles due to currently unaccessible experimental techniques. Taking a free electron as an example, the frequency and the amplitude of ZB is on the order of $10^{21} \mathrm{~Hz}$ and $10^{-3} \AA$, respectively, and the electric field gradients requirement for observing KT is on the order of $10^{18} \mathrm{~V} / \mathrm{m}$. All those conditions are out of reach in current technologies.

However, we will see that ultracold atoms loaded in some optical lattices or subject to certain synthetic gauge fields behave as the relativistic particles under suitable conditions. Furthermore, cold atom simulators offer us rather more degrees of freedom to control the relativistic quasiparticles, such as the dimensions, the effective mass and the effective speed of light. Associated with the controllable atomic interactions and disorder, these systems provide us an ideal platform to investigate the interesting relativistic effects such as the never-before-seen ZB and KT for free particles. Although the original Dirac equation is specific to fermions with spin $1 / 2$, not bosons with an integer spin, we will see that one can still simulate the Dirac equation with bosons: a pseudo-spin $1 / 2$ can be introduced to the bonons and the dynamics of such pseudo-spin should be described by the Dirac equation.

In this article, we review the recent theoretical investigation and experimental proposals in quantum simulation of the Dirac equation and some related effects by using ultracold neutral atoms. This review is organized as follows. In Section II, we introduce the realization and detection of the two and three-dimensional relativistic quasiparticles by using cold atoms in OLs with a wide range of structures. The realization of quantum anomalous Hall effect (QAHE) is also briefly introduced. In Section III, we introduce another kind of proposals for simulating the tunable Dirac equation that are based on generating effective gauge fields on bulk atomic gases. The simulated Dirac equations do not need OLs and thus operates in a continuous regime. In Section IV, we show that one can observe the well-known ZB and KT by using cold atoms with the schemes reviewed in Section III. Furthermore, we show that an exotic macroscopic KT described by the nonlinear Dirac equation (NLDE) exhibits. Finally, in Section V, we give our summary and conclusion of this review, together with some prospects on the quantum simulation of relativistic effects and related fields in physics.

\section{SIMULATION OF DIRAC EQUATION WITH ULTRACOLD ATOMS IN OL SYSTEMS}

In this section, we first review that ultracold neutral fermionic atoms in a honeycomb (hexagonal) OL, similar with electrons in graphene, can mimic 2D massless and massive Dirac fermions [41-47]. Then we demonstrate that, it is also possible to simulate 2D Dirac fermions in a so-called $\mathcal{T}_{3}$ OL [48,49], a line-centered-square (LCS) OL [50], and even in a simple square OL combing with a synthetic gauge potential [51-58]. Furthermore, we show that 3D Dirac fermions may be realized with cold atoms properly loaded in some cubic OLs [59-61]. The realization of quantum anomalous Hall effect (QAHE) associated to the parity anomaly of $2 \mathrm{D}$ Dirac fermions is also briefly introduced. In words, the emergent relativistic Dirac fermions in these OL systems originate from the single-particle band dispersion, which provides the Dirac cones in the Brillouin zone. Some lattices with symmetric structures like hexagonal, $\mathcal{T}_{3}$ and LCS in the real space, may directly support the needed structures of the momentum space; the simple square lattice may 
fail, and thus needs the aid of external fields. The nearhalf-filling condition allows the Fermi level being close to the Dirac points, and in the long wavelength limitation, the dynamics of the cold atoms are described well by the relativistic theories. The OLs allow one to tune the tunneling probabilities from site to site as well as the atomic interactions, and may make the relativistic quasiparticles realizable and controllable in these svstems.

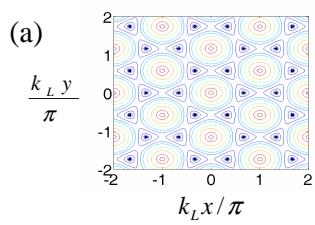

(c)

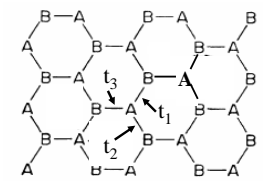

(e)
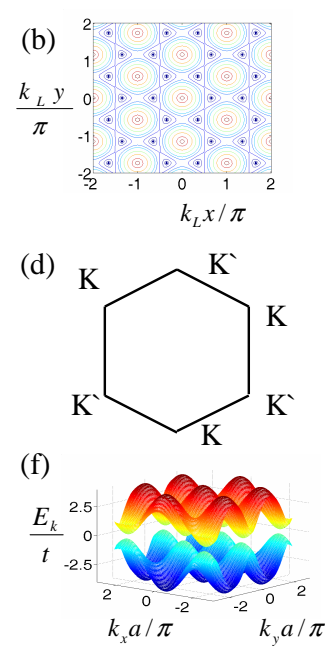

Fig. 1 The honeycomb OLs. (a),(b) The contours with three potentials described in Eq. (1). The minima of the potentials are denoted by the solid dots. All $V_{j}^{0}$ are the same in (a), and $V_{1}^{0}=$ $V_{2}^{0}=0.91 V_{3}^{0}$ in (b). (c) Decomposition of the hexagonal lattice as two triangular sublattices $A$ and $B$ with anisotropic tunnelling. (d) The Brillouin zone of the hexagonal lattice. The dispersion relations are shown in (e) for $\beta=1$ (gapless state) and (f) for $\beta=2.5$ (gapped state). Reproduced from Ref. [41], Copyright (C) 2007 the American Physical Society.

\section{A. Simulation of $2 \mathrm{D}$ relativistic Dirac fermions}

The graphene material, formed with a single layer of carbon atoms $[35,36]$ with its emergent massless Dirac fermions, has recently attracted strong interest in condensed-matter physics. One topic naturally arises is to mimic the graphene and the relativistic quasiparticles with cold atom in a similar 2D hexagonal lattice [41-47]. In addition to the graphene-type lattice, it is also interesting to search other proposals for quantum simulation of 2D Dirac equation with cold atoms in OLs of other structures [48-58].

\section{Mimic graphene: Cold atoms in a honeycomb $O L$}

Simulating Dirac equations with cold atoms loaded in a honeycomb OL was proposed in the first time in Ref. [41].
We review this proposal in this section. Considering single-component fermionic atoms (e.g., spin-polarized atoms ${ }^{40} \mathrm{~K},{ }^{6} \mathrm{Li}$, etc.) in a two-dimensional ( $x$ - $y$ plane) hexagonal OL formed with three detuned standing-wave lasers [62]

$$
V(x, y)=\sum_{j=1,2,3} V_{j}^{0} \sin ^{2}\left[k_{L}\left(x \cos \theta_{j}+y \sin \theta_{j}\right)+\frac{\pi}{2}\right]
$$

where $\theta_{1}=\pi / 3, \theta_{2}=2 \pi / 3, \theta_{3}=0$, and $k_{L}$ is the optical wave vector. It is easy to tune the potential barriers $V_{j}^{0}$ by varying the laser intensities along different directions to form a standard hexagonal lattice for $V_{1}^{0}=V_{2}^{0}=V_{3}^{0}$, and a hexagonal lattice but with a finite anisotropy for different $V_{j}^{0}$ as shown in Fig. 1(a) and 1(b), respectively. A hexagonal lattice consists of two sublattices denoted by $A$ and $B$, as shown in Fig. 1(c). For single-component fermionic atoms, the atomic collisions are negligible at low temperatures. The tight-binding Hamiltonian of the system is then given by

$$
\mathcal{H}=-\sum_{\langle i, j\rangle} t_{i j}\left(a_{i}^{\dagger} b_{j}+\text { H.c. }\right)
$$

where $\langle i, j\rangle$ represents the neighboring sites, $a_{i}$ and $b_{j}$ denote the fermionic mode operators for the sublattices $\mathrm{A}$ and $\mathrm{B}$, respectively. The tunneling rates $t_{i j}$ depend on the tunneling directions in an anisotropic hexagonal lattice, and we denote them as $t_{1}, t_{2}, t_{3}$ corresponding to the three different directions as shown in Fig. 1(c). For simplicity, we assume $t_{1}=t_{2}=t$ and $t_{3}=\beta t$ with $\beta$ being the anisotropy parameter. As the atomic tunneling rate in an OL is exponentially sensitive to the potential barrier, this control provides an effective method to control the anisotropy of the atomic tunneling by laser intensities. The first Brillouin zone of this system has also a hexagonal shape in the momentum space with only two of the six corners in Fig. 1(d) are inequivalent, corresponding to two different sites $A$ and $B$ in each cell in the real hexagonal lattice, usually denoted as $K$ and $K^{\prime}$. One can choose $\mathbf{K}=(2 \pi / a)(1 / \sqrt{3}, 1)$ and $\mathbf{K}^{\prime}=-\mathbf{K}$, where $a=2 \pi /\left(\sqrt{3} k_{L}\right)$ is the lattice spacing. Taking a Fourier transform $a_{i}^{\dagger}=(1 / \sqrt{N}) \sum_{\mathbf{k}} \exp \left(i \mathbf{k} \cdot \mathbf{A}_{i}\right) a_{\mathbf{k}}^{\dagger}$ and $b_{j}^{\dagger}=(1 / \sqrt{N}) \sum_{\mathbf{k}} \exp \left(i \mathbf{k} \cdot \mathbf{B}_{j}\right) a_{\mathbf{k}}^{\dagger}$, where $\mathbf{A}_{i}\left(\mathbf{B}_{j}\right)$ represents the position of the site in sublattice $A(B)$ and $N$ is the number of sites of the sublattice, the Hamiltonian (2) can be diagonalized and the eigenvalues have the expression [41]

$$
E_{\mathbf{k}}= \pm t \sqrt{2+\beta^{2}+2 \cos \left(k_{y} a\right)+4 \beta \cos \left(\sqrt{3} k_{x} a / 2\right) \cos \left(k_{y} a / 2\right)}
$$


As plotted in Fig. 1(e) and 1(f), there are two branches of the dispersion relation, corresponding to the \pm sign in Eq. (3). When $0<\beta<2$, the two branches touch each other, and around the touching points there appears a Dirac cone structure. One has the same standard Dirac cones as the graphene material with $\beta=1 \quad[35,63,64]$, and the cones squeeze in the $x$ or $y$ direction as $\beta$ deviates from 1 , but they still touch each other. When $\beta>2$, a finite energy gap $\Delta_{g}=|t|(\beta-2)$ appears between the two branch. So, across the point $\beta=2$, the topology of the Fermi surface changes, corresponding to a quantum phase transition without any usual symmetry breaking [65]. Such topological phase transition associated with pair production (annihilation) events has been investigated in Ref. [66]. The evolution of the Dirac points in the hexagonal lattice by varying the asymmetry hopping and the resulting phase transition was also studied in Ref. [44]. With this phase transition, the system changes its behavior from a semimetal to an insulator at the half filling case (means one atom per cell; note that each cell has two sites). Around the half filling, the Fermi surface is close to the touching points, and one can expand the momentum $\mathbf{k}$ around one of the touching points $\mathbf{K} \equiv\left(k_{x}^{0}, k_{y}^{0}\right)$ as $\left(k_{x}^{0}, k_{y}^{0}\right)=\left(k_{x}^{0}+q_{x}, k_{y}^{0}+q_{y}\right)$. Up to the second order of $q_{x}$ and $q_{y}$, the dispersion relation (3) becomes

$$
E_{\mathbf{q}}= \pm \sqrt{\Delta_{g}^{2}+v_{x}^{2} q_{x}^{2}+v_{y}^{2} q_{y}^{2}}
$$

where $\Delta_{g}=0, v_{x}=\sqrt{3} \beta t a / 2$, and $v_{y}=t a \sqrt{1-\beta^{2} / 4}$ for $0<\beta<2 ; \Delta_{g}=|t|(\beta-2), v_{x}=t a \sqrt{3 \beta / 2}$, and $v_{y}=t a \sqrt{\beta / 2-1}$ for $\beta>2$. This simplified dispersion relation $E_{\mathbf{q}}$ is actually a good approximation (named as long wavelength approximation) as long as $q_{x}, q_{y} \lesssim 1 / 2 a$. Compared with the standard energy-momentum relation for the relativistic Dirac particles, here $\Delta_{g}$ and $v_{x, y}$ take the meaning of rest energy and the velocity of light respectively. The wave function for the quasiparticles around the half filling then satisfies the Dirac equation $i \hbar \partial_{t} \Psi=\mathcal{H}_{D} \Psi$, where the relativistic Hamiltonian $\mathcal{H}_{D}$ is given by

$$
\mathcal{H}_{D}=v_{x} \sigma_{x} p_{x}+v_{y} \sigma_{y} p_{y}+\Delta_{g} \sigma_{z}
$$

where $\sigma_{x, y, z}$ are the three Pauli matrices.

Through an analogy to the graphene physics, we have shown that by controlling the lattice anisotropy, one can realize both massive and massless Dirac fermions and observe the phase transition between them. This proposal was recently proved to be experimentally feasible in Ref. [45], where the temperature requirement and crit- ical imperfections in the laser configuration are considered in detail. Even in the presence of a harmonic confining potential, the Dirac points are also found to survive [67]. In the presence of atomic interactions, the many-body physics of Dirac particles in graphene-type lattices, such as novel BCS-BEC crossover [42], topological phase transition between gapless and gapped superfluid [47] and even charge and bond ordered states with $p$-orbital band of lattices [43,68], have been investigated. Notably, the realization of ultracold quantum gases in a hexagonal OL was reported in a very recent experiment [69], which paves the important way to mimic the relativistic Dirac fermions and the aforementioned beyond-graphene physics with controllable systems.

\section{Pseudospin-1 massless Dirac fermions: Atoms in a $\mathcal{T}_{3} /$ line-centered-square $O L$}

It is interesting to note that cold atoms in OLs with other structures of symmetries can also simulate the $2 \mathrm{D}$ relativistic Dirac fermions. One of such examples was proposed in Ref. [48]. It showed that atoms trapped in the $\mathcal{T}_{3}$ lattice can behave as the massless Dirac fermions with pseudospin $S=1$, instead of $S=1 / 2$ for those in the hexagonal lattice. The so-call $\mathcal{T}_{3}$ lattice, illustrated in Fig. 2(a), has a unit cell with three different lattice sites, one sixfold coordinated site $\mathrm{H}$ and two threefold coordinated sites $\mathrm{A}$ and $\mathrm{B}$.

The $\mathcal{T}_{3}$ OL can be experimentally realized through three counterpropagating pairs of laser beams with the same wavelength $3 / 2 a$ and linearly polarized with the electrical field in the $x-y$ plane, which are similar with the laser setup for creating a hexagonal OL. Given a polarization of a pair of lasers on the $y$ axis, the other two pairs are obtained by rotating $2 \pi / 3$ around the $\mathrm{z}$ axis. The Schrödinger equation for the $\mathcal{T}_{3}$ OL filled with fermionic atoms in the tight-binding approximation is given by

$$
\begin{aligned}
& E \Psi_{H}\left(\mathbf{R}_{H}\right)=-t \sum_{j} \Psi_{A}\left(\mathbf{R}_{H}+\tau_{j}\right)+\Psi_{B}\left(\mathbf{R}_{H}+\tau_{j+1}\right),(6 \mathrm{a}) \\
& E \Psi_{\alpha}\left(\mathbf{R}_{\alpha}\right)=-t \sum_{j} \Psi_{H}\left(\mathbf{R}_{\alpha}-\tau_{j}\right), \quad \alpha \in\{A, B\} . \quad(6 \mathrm{~b})
\end{aligned}
$$

Here $\Psi_{\alpha}\left(\mathbf{R}_{\alpha}\right)$ is the amplitude of the wave function on sublattice $\alpha(=A, H, B)$, and the $\tau_{j}$ connect nearest neighbors. Solving the Eq. (6), one can obtain the energy-momentum relationship

$$
\begin{aligned}
& E_{0}(\mathbf{k})=0 \\
& E_{ \pm}(\mathbf{k})= \pm t \sqrt{6+4\left\{\cos \left[\left(\mathbf{v}_{1}-\mathbf{v}_{2}\right) \cdot \mathbf{k}\right]+\cos \left[\mathbf{v}_{1} \cdot \mathbf{k}\right]+\cos \left[\mathbf{v}_{2} \cdot \mathbf{k}\right\}\right]}
\end{aligned}
$$


the near half-filling case, one arranges the component $\alpha=\{A, H, B\}$ into a pseudospin triplet and expands the momentum $\mathbf{k}$ in the vicinity of $\mathbf{K}$, and thus obtains the effective Hamiltonian of Dirac-Weyl form given by

$$
\mathcal{H}_{D W}=v_{F} \mathbf{S} \cdot \mathbf{p}
$$

which describes the massless Dirac fermions with total pseudospin $S=1$. Here $v_{F}=3 t a / \sqrt{2}$ is the Fermi velocity, $\mathbf{p}$ is the momentum operator in the $x y$ plane, and the pseudospin vector operator $\mathbf{S}=\left(S_{x}, S_{y}, S_{z}\right)\left(S_{x, y, z}\right.$ are $3 \times 3$ matrices which satisfy angular momentum commutation relations) reflected the three inequivalent lattice sites per unit cell in $\mathcal{T}_{3}$. A comparison on the topological properties between the hexagonal lattice and the $\mathcal{T}_{3}$ lattice was given in Ref. [49], where the former is shown to be topological insulator and the latter to be trivial insulator in the framework of quantum spin Hall states.
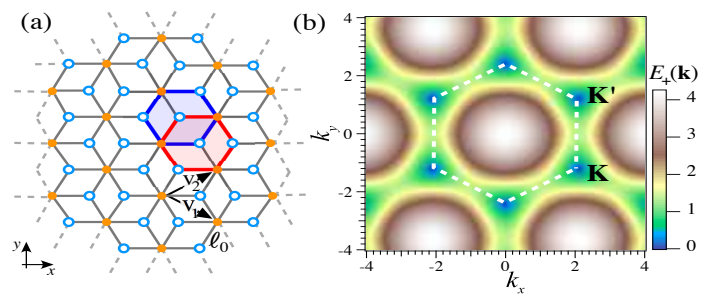

Fig. 2 (a) The $\mathcal{T}_{3}$ lattice. It is characterized by translation vectors $\mathbf{v}_{1}=(3 / 2 ;-\sqrt{3} / 2) a$ and $\mathbf{v}_{2}=(3 / 2 ; \sqrt{3} / 2) a$. Open circles mark the two sublattices $A$ and $B$, forming a hexagonal lattice. Solid circles mark the hub sites $H$ forming a (larger) triangular lattice. (b) Contour plot of $E_{+}(\mathbf{k})$ in units of the hopping energy $t$, cf. Eq. (7b). The dashed hexagon defines the first Brillouin zone, $\mathbf{K}=2 \pi a^{-1}(1 / 3 ;-\sqrt{3} / 9)$ and $\mathbf{K}^{\prime}=2 \pi a^{-1}(1 / 3 ; \sqrt{3} / 9)$ are two nonequivalent Dirac points. Reproduced from Ref. [48], Copyright (C) 2009 the American Physical Society.
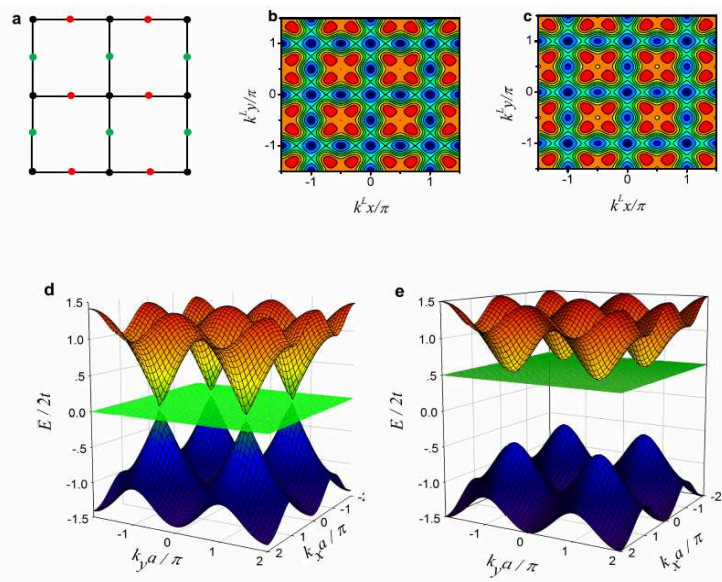

Fig. 3 (a) Schematic illustration of the LCS lattice, energy contour of the optical potential in Eq. (9) with (b) $V_{1}=2 V_{2}$ and (c) $V_{1}=2.2 V_{2}$, and dispersion relation on the LCS lattice with (d) $\Delta_{g}^{\prime}=0$ and (e) $\Delta_{g}^{\prime}=t$. Reproduced from Ref. [50], Copyright (C) 2010 the American Physical Society.

Another example of quantum simulation of 2D massless Dirac fermion was proposed to be realized in a LCS
OL [50]. The massless Dirac fermions there also have pseudospin-1, and may exhibit a perfect all-angle KT (we will discuss KT in the Section IV), i.e., the barrier is completely transparent for all incident angles [50]. The LCS lattice is schematically illustrated in Fig. 3(a), with three sublattices denoted by the black (sites $A$ ), red (sites $B$ ), and green (sites $C$ ) points, respectively. The LCS lattice can be realized in the ultracold atomic system by applying six detuned standing-wave laser beams, four of which are applied along the $\mathbf{e}_{x}$ and $\mathbf{e}_{y}$ directions with optical wave vectors $k_{L}$ and $2 k_{L}$, respectively; the other two are applied along the $\left(\mathbf{e}_{x}+\mathbf{e}_{y}\right) / \sqrt{2}$ directions with optical wave vector $2 k_{L}$ and relative phase $\pi / 2$, respectively. Thus the whole optical potential is

$$
\begin{aligned}
V(x, y)= & V_{1}\left[\sin ^{2}\left(k_{L} x\right)+\sin ^{2}\left(k_{L} y\right)+\sin ^{2}\left(2 k_{L} x\right)\right. \\
& \left.+\sin ^{2}\left(2 k_{L} y\right)\right]+V_{2}\left\{\sin ^{2}\left[k_{L}(x+y)+\frac{\pi}{2}\right]\right. \\
& \left.\cdot \sin ^{2}\left[k_{L}(x-y)+\frac{\pi}{2}\right]\right\}
\end{aligned}
$$

with tunable potential amplitudes $V_{1}$ and $V_{2}$, and lattice constant $a=\pi / k_{L}$. Two examples of energy contours of LCS OL are plotted in Fig. 3(b) and 3(c), in which the potential minima are marked with the black and blue points. For $V_{1}=2 V_{2}, V(x, y)$ is the same at sites $A$ - $C$ so that their site energies $\epsilon_{A}=\epsilon_{B}=\epsilon_{C}$. While for $V_{1} \neq 2 V_{2}, \epsilon_{B}=\epsilon_{C} \neq \epsilon_{A}$ and one may set $\epsilon_{B}=$ $-\epsilon_{A}=\Delta_{g}^{\prime}$ for the symmetry consideration. The Hamiltonian of LCS lattice $\mathcal{H}_{L C S}=\sum_{i} \epsilon_{i} c_{i}^{\dagger} c_{i}+t \sum_{\langle i, j\rangle} c_{i}^{\dagger} c_{j}$ can be diagonalized with three branches through a similar procedure for Eq. (2). One is a flat band with energy $E_{0}=\Delta_{g}^{\prime}$, and the other two dispersive bands are $E_{ \pm}= \pm \sqrt{\Delta_{g}^{\prime 2}+4 t^{2}\left[\cos ^{2}\left(k_{x} a / 2\right)+\cos ^{2}\left(k_{y} a / 2\right)\right]}$. Typical energy bands are shown in Fig. 3(d) for $\Delta_{g}^{\prime}=0$ and Fig. $3(\mathrm{e})$ for $\Delta_{g}^{\prime}=t$.

At $\Delta_{g}^{\prime}=0$, only one nonequivalent Dirac point appears at $\mathbf{K}=(\pi / a, \pi / a)$. In the vicinity of the Dirac cone, the ultracold atoms behave as the massless Dirac fermions with pseudospin-1, described by Eq. (8) with $v_{F}=t a$ in this case. Interestingly, the massless Dirac fermions in the HSC lattice are topologically different from those in the hexagonal and $\mathcal{T}_{3}$ OLs presented previously because there are not two nonequivalent Dirac cones but a single one in the first Brillouin zone. Note that the solidstate material with a single (or odd number) Dirac cone, named as strong topological insulator, has attract considerable attention recently [37]. Besides, the flat band with Dirac cones meeting at the same energy, which means the particle can have an infinite effective mass (flat band) or a zero effective mass (Dirac fermions), may result in interesting atom dynamics in OLs, such as different behaviors of atomic localization for bosons and fermions [70]. 


\section{Dirac fermions in a square $O L$ with a gauge field}

The massless Dirac fermions can also be simulated by using ultracold atoms in a simple square OL subjected to certain light-induced synthetic gauge fields (which will be addressed in Section III). The synthetic fields are necessary because the energy bands of a single square lattice do not exhibit the Dirac cone structure. (a)

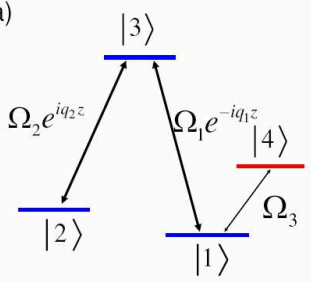

(c)

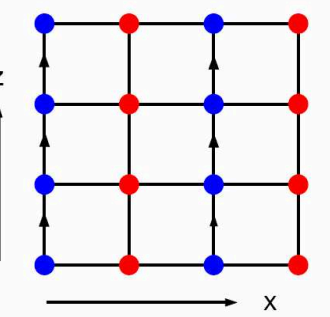

(b)

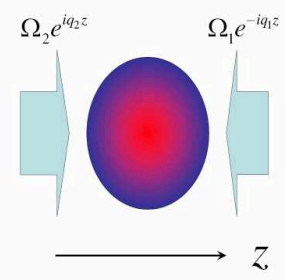

(d)

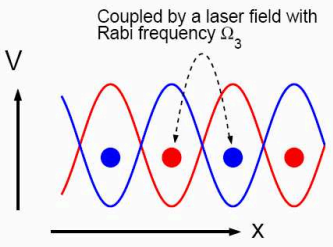

Fig. 4 (a) The atomic levels and the interactions between atoms and laser fields. (b) Schematic representation of the experimental setup with the two laser beams incident on the cloud of atoms. (c) Schematic of the square optical lattice and the designed phase factor (denoted by arrows). (d) The scheme of overlapping the two state-selective optical lattices. Reproduced from Ref. [52], Copyright (C) 2009 the American Physical Society.

The first scheme along this line was proposed in Ref. [52], where the fermionic atoms are loaded in a square OL subjected to a U(1) gauge field generated by laser-atom interactions. As shown in Fig. 4(a) and (b), the noninteracting atoms of mass $m$ with four levels in the $x-z$ plane are coupled with three laser beams. The ground state $|1\rangle$ is coupled to the excited state $|3\rangle$ via a laser field with the corresponding Rabi frequency $\Omega_{1} e^{-i q_{1} z}$ and the state $|2\rangle$ is coupled to the excited state $|3\rangle$ via a laser field with the corresponding Rabi frequency $\Omega_{2} e^{i q_{2} z}$. The cold atoms are trapped in two stateselective optical potentials as shown in Fig. 4(c) and (d), say atoms with internal states $|1\rangle,|2\rangle$, and $|3\rangle$ are trapped in odd columns (sublattice $A$ ), while atoms with internal states $|4\rangle$ are trapped in even columns (sublattice $B)$. The two sublattices make up a $2 \mathrm{D}$ rectangular lattice with the lattice spacings $a_{x}$ and $a_{z}$, especially a 2D square lattice for $a_{x}=a_{z}$. In the basis $\{|1\rangle,|2\rangle,|3\rangle,|4\rangle\}$, the total Hamiltonian of this system can be written as

$\mathcal{H}_{S}=\hat{H}_{0}+\hbar\left(\begin{array}{cccc}V_{A} & 0 & \Omega_{1} e^{i q_{1} z} & \Omega_{3} \\ 0 & V_{A} & \Omega_{2} e^{-i q_{2} z} & 0 \\ \Omega_{1} e^{-i q_{1} z} & \Omega_{2} e^{i q_{2} z} & V_{A} & 0 \\ \Omega_{3} & 0 & 0 & V_{B}\end{array}\right)$

where $\hat{H}_{0}=-\frac{\hbar^{2}}{2 m} \nabla^{2}$ is kinetic energy operator, and $V_{A, B}$ are the two state-selective periodic potentials. Diagonalizing the Hamiltonian (10) yields two degenerate dark states (with eigenenergy $E=0$ )

$$
\begin{aligned}
& \left|\chi_{1}\right\rangle=\cos \theta|1\rangle-\sin \theta e^{i q z}|2\rangle, \\
& \left|\chi_{2}\right\rangle=|4\rangle,
\end{aligned}
$$

and two bright states, where $q=q_{1}+q_{2}$ and $\tan \theta=$ $\left|\Omega_{1}\right| /\left|\Omega_{2}\right|$. If the motions of the atoms that initially prepared in the dark state subspace satisfy the adiabatic condition, one can safely use the adiabatic approximation and reduce the Hamiltonian to

$$
\begin{aligned}
\hat{H}= & \int d^{2} r \hat{\Phi}_{1}^{\dagger}\left[\frac{1}{2 m}(-i \hbar \nabla-\mathbf{A})^{2}+V_{A}\right] \hat{\Phi}_{1} \\
& +\int d^{2} r \hat{\Phi}_{4}^{\dagger}\left[-\frac{\hbar^{2}}{2 m} \nabla^{2}+V_{B}\right] \hat{\Phi}_{4} \\
& +\hbar \Omega_{e} \int d^{2} r\left(\hat{\Phi}_{4}^{\dagger} \hat{\Phi}_{1}+\hat{\Phi}_{1}^{\dagger} \hat{\Phi}_{4}\right),
\end{aligned}
$$

in the second quantized form, using $\left\{\left|\chi_{1}\right\rangle,\left|\chi_{2}\right\rangle\right\}$ as the basis. Here $\hat{\Phi}_{i}(\mathbf{r})$ and $\hat{\Phi}_{i}^{\dagger}(\mathbf{r})$ are field operators corresponding to annihilating and creating an atom with the internal quantum state $|i\rangle$ at coordinate position $\mathbf{r}$ respectively, $\Omega_{e}=\Omega_{3} \cos \theta$ and the $\mathrm{U}(1)$ adiabatic gauge potential $\mathbf{A}=\hbar q \sin ^{2} \theta \mathbf{e}_{z}$. Taking the tight-binding limit, one can superpose the Bloch states to get Wannier functions $w_{a}\left(\mathbf{r}-\mathbf{r}_{\mathbf{i}}\right)$ and $w_{b}\left(\mathbf{r}-\mathbf{r}_{\mathbf{j}}\right)$ for sublattice $A$ and $B$, respectively. Expanding the field operator in the lowest band Wannier functions as

$$
\begin{aligned}
& \hat{\Phi}_{1}(\mathbf{r})=\sum_{m(\text { odd }), n} \hat{a}_{m, n} e^{\frac{i}{\hbar} \int_{0}^{\mathbf{r}_{m n}} \mathbf{A} \cdot d \mathbf{r}} w_{a}\left(\mathbf{r}-\mathbf{r}_{m n}\right), \\
& \hat{\Phi}_{4}(\mathbf{r})=\sum_{m(\text { even }), n} \hat{b}_{m, n} w_{b}\left(\mathbf{r}-\mathbf{r}_{m n}\right),
\end{aligned}
$$

and then substituting them into Eq. (12), one can rewrite the Hamiltonian as

$$
\begin{gathered}
\hat{H}=-t \sum_{[m(\text { odd }), n]} \hat{b}_{m+1, n+1}^{\dagger} \hat{b}_{m+1}+e^{i \gamma} \hat{a}_{m, n+1}^{\dagger} \hat{a}_{m, n} \\
+2 \hat{a}_{m, n}^{\dagger} \hat{b}_{m+1, n}+\text { H.c. },
\end{gathered}
$$

where $\gamma=2 \pi \hbar a_{z} \sin ^{2} \theta$ is the phase resulted from the adiabatic gauge potential. Here the isotropic atomic tunneling is assumed, which can be realized by well adjusting the intensities of laser beams. Diagonalizing the Hamiltonian (14) with $\gamma=\pi$ and $a_{x}=a_{z}=a$, one obtains the quasiparticle energy spectrum $E(\mathbf{k})=$ $\pm 2 t \sqrt{\cos ^{2}\left(k_{x} a\right)+\cos ^{2}\left(k_{z} a\right)}$, which exhibits two inequivalent Dirac points in the first Brillouin zone with one at $\mathbf{K}=(\pi / 2 a, \pi / 2 a)$. In the vicinity of the Dirac point $\mathbf{K}$, the atoms behave like the massless Dirac fermions described by the effective Dirac-like Hamiltonian

$$
\hat{\mathcal{H}}=\hbar v_{F}\left(\hat{p}_{x} \sigma_{x}+\hat{p}_{z} \sigma_{z}\right)
$$

where $v_{F}=2 t a / \hbar$ is the Fermi velocity of this system. 
The 2D massless Dirac fermions also emerge when ultracold fermionic atoms are trapped in a square OL subjected to a $\mathrm{U}(2)$ non-Abelian gauge field [56], that is to say, the phase factor $\gamma$ in Eq. (14) is replaced by a $2 \times 2$ matrix. This kind of non-Abelian OLs could be created by laser-assisted tunneling for atoms in optical superlattices [71-73]. They are characterized by a constant Wilson loops, and the single-particle spectrum may depict a complex structure termed as the Hofstadter "butterfly" [74]. In the proposal, the half-integer quantum Hall effect that has been observed in the graphene $[35,36]$ and the squeezed Landau vacuum due to anisotropic of external gauge filed are also investigated.

The external gauge filed can even be replaced by a time-dependent optical potential [53,54], which acts to shake the lattice, leading to the modification of effective tunneling strength. Such driven tunneling can be used to generate an artificial staggered magnetic field for atoms in a two-dimensional square optical lattice. For bosonic gas in this optical system, the zero-temperature phase diagram was shown to exhibit a finite-momentum superfluid phase with a quantized staggered rotational flux [53]. For fermionic gas, 2D massless Dirac fermions may also be provided [54]; and mixing both bosonic and fermionic atoms, one may even reach the strongly interacting regime for 2D Dirac fermions [55], which allows to investigate the transition between the Dirac and the Fermi liquid, and some correspondences with hightemperature cuprates.

Another model with a different time-dependent optical potential, which is also used to create an artificial magnetic field in cold atoms loaded on a square OL, was recently presented in Ref. [58]. The effective dynamics of low energy quasiparticles in that system can be described by a Dirac Hamiltonian for massless fermions with the unusual property that chiral symmetry is broken in the tunneling energy term, leading to splitting the doubly degenerate Dirac cones into two with tunable slopes. The two slopes describe two speeds of light for the relativistic quasiparticles.

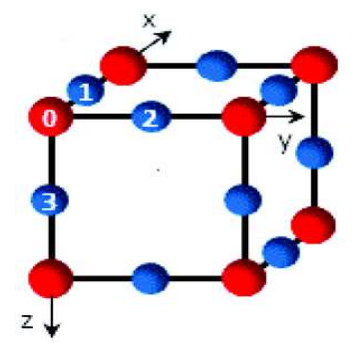

Fig. 5 A crystal cell of the edge-centered cubic lattice and the axis system adapted in this paper. One crystal cell includes four inequivalent lattice sites labeled by $0,1,2$ and 3, respectively. Reproduced from Ref. [59], Copyright (C) 2010 the American Physical Society.

\section{B. Simulation of $3 \mathrm{D}$ relativistic Dirac fermions}

The above mentioned work are all limited in 2D Dirac fermions, and the 3D ones in the OLs would be valuable to explore since the 3D Dirac fermions differ from 2D ones by transport properties, localization, etc. We review in the following that cold atoms loaded in an edge-centered cubic (ECC) OL can be used to simulate the 3D Diraclike fermions [59].

The ECC lattice as shown in Fig. 5 is the three dimensional counterpart of the LCS. In certain conditions (established by Eq. (19) below), the cold atoms on the lattice sites can be regarded approximately as the ground states of anisotropic 3D harmonic oscillators with energy $\epsilon_{g}^{(i)}=\frac{1}{2} \hbar \sum_{\nu=x, y, z} \omega_{\nu}^{i}$, where $\omega_{\nu}^{(i)}=\left[\partial_{\nu}^{2} V / m\right]_{\mathrm{i}}^{1 / 2}$ is the frequency of the oscillator on site $i$ along the axis $\nu$ with potential $V$ (see Ref. [59] for its expression). Substituting $V$ into the expression of $\omega^{i}$, yields

$$
\begin{aligned}
& \epsilon_{g}^{(0)}=E_{r}\left[\frac{3}{2} \sqrt{\alpha_{1}-4 \alpha_{2}+4 \alpha_{3}}\right], \\
& \epsilon_{g}^{(1)}=E_{r}\left[\sqrt{\alpha_{1}+4 \alpha_{3}}+\frac{1}{2} \sqrt{-\alpha_{1}+4 \alpha_{2}+4 \alpha_{3}}\right], \\
& \epsilon_{g}^{(3)}=\epsilon_{g}^{(2)}=\epsilon_{g}^{(1)},
\end{aligned}
$$

where $\alpha_{i}=A_{i} / E_{r}(i=1,2,3)$ are dimensionless parameters with $E_{r}=\hbar^{2}(2 \pi / a)^{2} / 2 m$ being the recoil energy. The $3 \mathrm{D}$ harmonic potentials on sites 0 and 1 are lifted by the bottom energies $V(0,0,0)=6 A_{2}$ and $V(a / 2,0,0)=A_{1}+2 A_{2}$ on site 0 and site 1 , respectively. The ground state energies of these sites are

$$
\begin{aligned}
& E_{g}^{(0)}=\epsilon_{g}^{(0)}+6 \alpha_{2} E_{r}, \\
& E_{g}^{(1)}=\epsilon_{g}^{(1)}+\left(\alpha_{1}+2 \alpha_{2}\right) E_{r} .
\end{aligned}
$$

Since the ground state energies on lattice sites 1, 2 and 3 are the same, we may address only one of them, such as $E_{g}^{(1)}$ in Eq. (17b). We limit ourself to the energy band formed by the ground states of the lattice sites. To this end, one has to tune the parameters $A_{1}, A_{2}$ and $A_{3}$ (or $\alpha_{1}, \alpha_{2}$ and $\alpha_{3}$ ) carefully to satisfy the following conditions

$$
\begin{aligned}
& \left|E_{g}^{(0)}-E_{g}^{(1)}\right| \ll 2 \min \left[\epsilon_{g}^{(0)}, \epsilon_{g}^{(1)}\right], \\
& \left|\epsilon_{g}^{(0)}+\epsilon_{g}^{(1)}\right| \ll 2 V_{j} .
\end{aligned}
$$

The parameter $V_{j}$ is the well depth that can be estimated as $V_{j}=[2 V(0,0, a / 4)-V(0,0,0)-V(0,0, a / 2)] / 2=A_{3}$. Equation 19(a) ensures that other energy levels of the oscillators on each sites are separated sufficiently far away and then the energy bands are formed by the ground states, and Eq. 18(b) guarantees that the calculated ground levels on all sites are much lower than the well depth so as to local levels can be formed and the ground energies can be evaluated by Eq. (16a)-(17b). One can estimate that the parameter region with $V_{1} / V_{2} \approx 4$ and $V_{3} \gtrsim 30 E_{r}$ fulfills the above condition. 
Under the above harmonic approximate conditions, the tight-binding Hamiltonian of the system in momentum space can be worked out as

$$
H_{k}=2 t\left(\begin{array}{cccc}
-\delta & \cos \Gamma_{x} & \cos \Gamma_{y} & \cos \Gamma_{z} \\
\cos \Gamma_{x} & \delta & 0 & 0 \\
\cos \Gamma_{y} & 0 & \delta & 0 \\
\cos \Gamma_{z} & 0 & 0 & \delta
\end{array}\right)
$$

in the basis $\{|0\rangle,|1\rangle,|2\rangle,|3\rangle\}$ with $|i\rangle$ being the single particle ground state on site $i$. Here the mid-point of the energy on sites 0 and 1 is chosen as the energy reference, $\Gamma_{x, y, z}=k_{x, y, z} a / 2$ with wave vector $k_{i}(i=x, y, z)$ along the direction $i, 2 \delta=E_{g}^{(1)}-E_{g}^{(0)}$ is the energy difference between site 1 and site 0 , and $t$ is the hopping constant. Diagonalizing $H_{k}$, one obtains the four band dispersions given by

$$
\begin{aligned}
& E_{1}=E_{2}=\delta, \\
& E_{ \pm}= \pm \sqrt{4 t^{2}\left(\cos ^{2} \Gamma_{x}+\cos ^{2} \Gamma_{y}+\cos ^{2} \Gamma_{z}\right)+\delta^{2}} .
\end{aligned}
$$

The first two dispersions are flat bands stick to the bottom of the upper band or the top of the lower band, depending on the sign of $\delta$. Note that 3D bulk flat band has not attracted much attention, in contract to previous work based on $1 \mathrm{D}$ or $2 \mathrm{D}$ model $[50,56,70]$. The last two dispersions possess the characteristic of Dirac particles near the only one Dirac point $\mathbf{K}=(\pi / a, \pi / a, \pi / a)$ at the corner of the first Brillouin zone, and they touch each other when $\delta=0$. At the vicinity of the Dirac point, the dispersion (21b) is approximately rewritten as

$$
\tilde{E}_{ \pm}= \pm \sqrt{m^{* 2} c^{* 4}+p^{2} c^{* 2}},
$$

where $p=\hbar k$ is the momentum with $k=\left(k_{x}^{2}+k_{y}^{2}+k_{z}^{2}\right)^{1 / 2}$ being the amplitude of $3 \mathrm{D}$ wave-vector, $c^{*}=a t / \hbar$ is the effective light speed, and $m^{*}=\hbar^{2} \delta /(a t)^{2}$ is the effective rest mass. The dispersion describes $3 \mathrm{D}$ massive Dirac fermions, and reduced to massless Dirac dispersion, i.e., $E_{ \pm}= \pm c^{*} p$, when $\delta=0$.

Apart from this scheme, it has been shown [60] that the $3 \mathrm{D}$ relativistic fermions can also be simulated with ultracold fermionic atoms in a 3D optical superlattice as an extension system of 2D non-Abelian OL in [56]. Interestingly, the $3 \mathrm{D}$ relativistic fermions simulated in this way are the so-call naive Dirac fermions in lattice gauge theories $[75,76]$, and thus provide a realization of the fermion doubling problem: Two massless Dirac fermions appear in the Brillouin zone, each of them has a different chirality, corresponding to the right and left-handed particles. Furthermore, by tuning the laser intensities, the system may allow to decouple the doublers from a single Dirac fermion through inverting their effective mass, i.e., a quantum simulation of Wilson fermions [77]. In this regime, the atomic gas corresponds to a phase of matter, 3D topological insulators, where Maxwell electrodynamics is replaced by axion electrodynamics $[37,78]$. The chirality symmetry breaking via mass terms is also crucial for simulating the Wess-Zumino supersymmetry model [34].

The 3D Dirac fermions using ultracold atoms are also proposed to be realized in a cubic optical lattice subjected to a synthetic frustrating magnetic field $\vec{B}=$ $\pi \phi_{0}(1,1,1)$ with $\phi_{0}=\hbar / 2 m a^{2}$ [61]. The tight-binding Hamiltonian of the system can be described by

$$
H=-t \sum_{\langle i, j\rangle}\left(c_{i}^{\dagger} e^{-i A_{i j}} c_{j}+\text { h.c. }\right)
$$

where the phase factor is $A_{i j}=\frac{m}{\hbar} \int_{i}^{j} \vec{A} \cdot d \vec{l}$, with the vector $\vec{A}=\pi(0, x-y, y-x)$ since $\vec{B}=\operatorname{rot} \vec{A}$. Diagonalization of Hamiltonian (22) yields the dispersion as

$$
E_{ \pm}= \pm 2 t \sqrt{\left(\cos ^{2} k_{x} a+\cos ^{2} k_{y} a+\cos ^{2} k_{z} a\right)},
$$

which exhibit two inequivalent Dirac points again. Around the Dirac points, the atoms behave like the 3D massless Dirac fermions. It is also shown to be possible to give mass to the Dirac fermions by coupling the ultracold atoms to a Bragg pulse, and to obtain a dimensional crossover from $3 \mathrm{D}$ to $2 \mathrm{D}$ Dirac fermions by varying the anisotropy of the lattice.
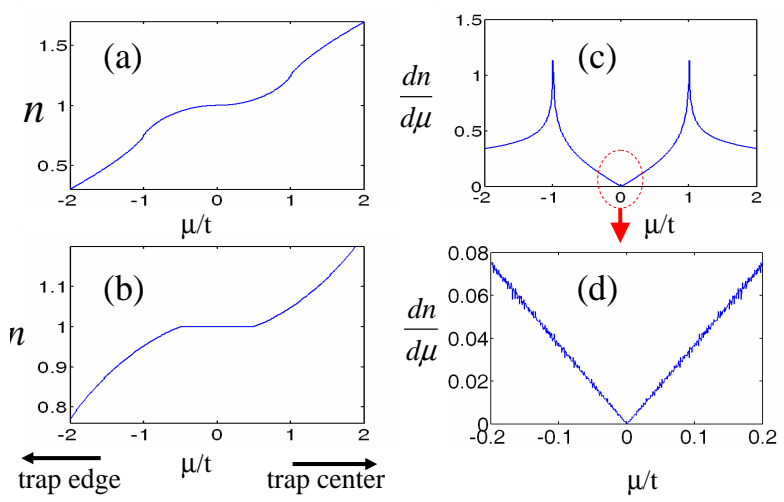

Fig. 6 The number density of atoms $n$ per unit cell of the hexagonal lattice as a function of the chemical potential $\mu$ (corresponding to a re-scaled atomic density profile in a trap) for (a) $\beta=1$, and (b) $\beta=2.5$. A plateau with a width $2 \beta-4$ appears for the latter case which corresponds to the case when the chemical potential sweeps inside the energy gap. (c) The derivative $d n / d \mu$ as a function of the chemical potential $\mu$ for $\beta=1$. (d) An enlarged part of $d n / d \mu$ at the vicinity of $\mu=0$. The linearity of the curve shows the linear dispersion relation for the quasiparticles. Reproduced from Ref. [41], Copyright (C) 2007 the American Physical Society.

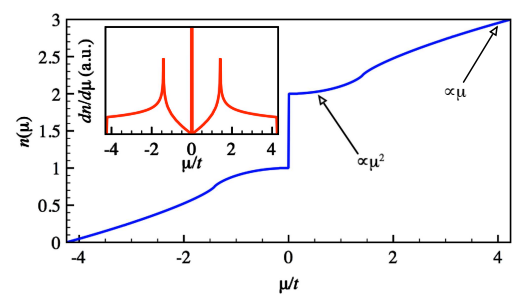

Fig. 7 The number of atoms $n$ per unit cell in $\mathcal{T}_{3}$ OLs at zero temperature as a function of $\mu / t$. Inset: density of states, $d n / d \mu$, versus $\mu / t$. Reproduced from Ref. [48], Copyright (C) 2009 the American Physical Society. 


\section{Detection of the Dirac quasiparticles}

We have shown that cold atoms trapped in OLs with a wide range of structures can be used to simulate the Dirac equation, and the quasiparticles in these systems behave as the relativistic ones. Another important issue, however, is to experimentally verify the existence of these relativistic quasiparticles. The widely used detection technique based on the transport measurements for the condensed-matter materials is typically not available for the atoms, and nevertheless, there are some specific detection methods for the trapped atomic gas. For simplicity, we focus on the aforementioned hexagonal OL system [41], and in the following we review two different methods to confirm the relativistic quasiparticles and the phase transition between massless and massive ones [41]: the density profile measurement [79] and the Bragg spectroscopy [80]. However, the detection methods can be extended to other systems without loss of generality.

Density profile measurement.-The density profile of the trapped atoms can be measured through the timeof-flight imaging with the light absorption [79]. Free fermions expand with ballistic motion and from the final measured absorption images, one can reconstruct the initial real-space density profile of the trapped gas. Under the local density approximation, the local chemical potential varies with the radial coordinate by $\mu=\mu_{0}-V(\mathbf{r})$, where $\mu_{0}$ is the chemical potential at the trap center and $V(\mathbf{r})=m \omega^{2} \mathbf{r}^{2} / 2$ is the global harmonic trapping potential. At temperature $T$, the atomic density is given by

$$
n(\mu)=\frac{1}{S_{0}} \int f\left(k_{x}, k_{y}, \mu\right) d k_{x} d k_{y},
$$

where $S_{0}=8 \pi^{2} / \sqrt{3} a^{2}$ is the area of the first Brillouin zone of the hexagonal lattice, and $f\left(k_{x}, k_{y}, \mu\right)=$ $1 /\left\{\exp \left[\left(E_{\mathbf{k}}-\mu\right) / T\right]+1\right\}\left(E_{\mathbf{k}}\right.$ in Eq. $\left.(3)\right)$ is the Fermi-Dirac distribution. At low temperature $(T \sim 0)$, this density profile $n(\mu)$ is shown in Fig. 6(a) and 6(b) for the parameters with massless and massive Dirac quasiparticles, respectively. Clearly, a plateau at the atom density $n=1$ in the density profile appears for the gapped phase with massive Dirac fermions; but there is no such a plateau for the case with massless Dirac fermions. So the plateau is associated with massive quasiparticles, and its emergence provides an unambiguous signal for the quantum phase transition between the two cases. Furthermore, the linear dispersion relation for the massless Dirac fermions can be confirmed by the derivative $d n / d \mu$ since one has $\frac{\partial n}{\partial \mu}=\frac{4 \pi}{v^{2} S_{0}}|\delta \mu|$ around the Dirac cone for $\beta=1$ and $v_{x}=v_{y}=v$. As shown in Fig. 6(c) and (d), $\frac{\partial n}{\partial \mu}$ is linearly proportional to $\delta \mu$ indeed at the vicinity of the half filling. So experimentally, from the measured density profile $n(\mu)$, one can determine its slope, which signals the linear dispersion relation, to confirm the massless Dirac fermions.

This method is also available for the detection of massless Dirac fermion in $\mathcal{T}_{3}$ OLs [48]. The similar results are shown in Fig. 7, but with a sharp jump in the atomic density at $\mu=0$ due to the contribution from the highly degenerate flat band.

Bragg spectroscopy.-The Bragg spectroscopy can provide an alternative and complementary method to confirm the linear dispersion relation for the massless Dirac fermions and the energy gap for the massive ones [41]. In Bragg spectroscopy [80], one shines two laser beams on the atomic gas as shown in Fig. 8(a). By fixing the angle between the two beams (thus fixing the relative momentum transfer $\mathbf{q}=\mathbf{k}_{2}-\mathbf{k}_{1}$, where $\mathbf{k}_{i}$ denotes the wave vector of each laser beam), one can measure the atomic (or photonic) transition rate by scanning the laser frequency difference $\omega=\omega_{2}-\omega_{1}$. From the Fermi's golden rule, this transition rate basically measures the following dynamical structure factor [80]

$$
S(\mathbf{q}, \omega)=\sum_{\mathbf{k}_{1}, \mathbf{k}_{2}}\left|\left\langle f_{\mathbf{k}_{2}}\left|H_{B}\right| i_{\mathbf{k}_{1}}\right\rangle\right|^{2} \delta\left[\hbar \omega-E_{f \mathbf{k}_{2}}+E_{i \mathbf{k}_{1}}\right]
$$

where $H_{B}=\sum_{\mathbf{k}_{1}, \mathbf{k}_{2}} \Omega e^{i \mathbf{q} \cdot \mathbf{r}}\left|i_{\mathbf{k}_{1}}\right\rangle\left\langle f_{\mathbf{k}_{2}}\right|+h . c$. is the lightatom interaction Hamiltonian, and $\left|i_{\mathbf{k}_{1}}\right\rangle$ and $\left|f_{\mathbf{k}_{2}}\right\rangle$ denote the initial and the final atomic states with the energies $E_{i \mathbf{k}_{1}}$ and $E_{f \mathbf{k}_{2}}$ and the momenta $\mathbf{k}_{1}$ and $\mathbf{k}_{2}$, respectively. At the half filling, the excitations are dominantly around the touching point, and we can use the approximate dispersion relation in Eq. (4). For the isotropic case $(\beta=1)$ with massless Dirac Fermions, $S(q, \omega)$ has the expression

$$
S(q, \omega)= \begin{cases}0, & \left(\omega \leq \omega_{r}\right) \\ \frac{\pi \Omega^{2}}{8 v_{F}} \frac{2 q_{r}^{2}-q^{2}}{\sqrt{q_{r}^{2}-q^{2}}}, & \left(\omega>\omega_{r}\right)\end{cases}
$$

where $\omega_{r}=q v_{F} / \hbar(q \equiv|\mathbf{q}|)$ and $q_{r}=\hbar \omega / v_{F}$. This dynamical structure factor is shown in Fig. 8(b). Note that in this case, the lower cutoff frequency $\omega_{r}$ is linearly proportional to the momentum difference $q$, and $\omega_{r}$ vanishes when $q$ tends to zero. The ratio between $\omega_{r}$ and $q$ gives the Fermi velocity $v_{F}$, an important parameter as the analogy of the light velocity for conventional relativistic particles. For the anisotropic case with $\beta>2$, the spectrum in Eq. (4) becomes quadratic with $E \approx \pm\left(\Delta+\hbar^{2} q_{x}^{2} / 2 m_{x}+\hbar^{2} q_{y}^{2} / 2 m_{y}\right)$ for small momentum transfer $\mathbf{q}$, where the effective mass $m_{x, y}=\hbar^{2} \Delta / v_{x, y}^{2}$. The dynamical structure factor in this non-relativistic limit becomes

$$
S(q, \omega)= \begin{cases}0, & \left(\omega \leq \omega_{c}^{x, y}\right) \\ \frac{\pi \Omega^{2} \Delta}{2 v_{x} v_{y}}, & \left(\omega>\omega_{c}^{x, y}\right)\end{cases}
$$

where $\omega_{c}^{x, y}=2 \Delta+\hbar^{2} q_{x, y}^{2} / 4 m_{x, y}$. Its form is shown in Fig. 8(b). The lower cutoff frequency $\omega_{c}^{x, y}$ in this case does not vanish as the momentum transfer goes to zero. This distinctive difference between the dynamical structure factors in Eqs. (26) and (27) can be used to distinguish the cases with massive or massless Dirac fermions. From the variation of the cutoff frequency $\omega_{c}^{x, y}$ as a function of the momentum transfer $q_{x, y}$, one can also experimentally figure out the important parameters such as the energy gap $\Delta$ and the effective masses $m_{x}$ and $m_{y}$. 


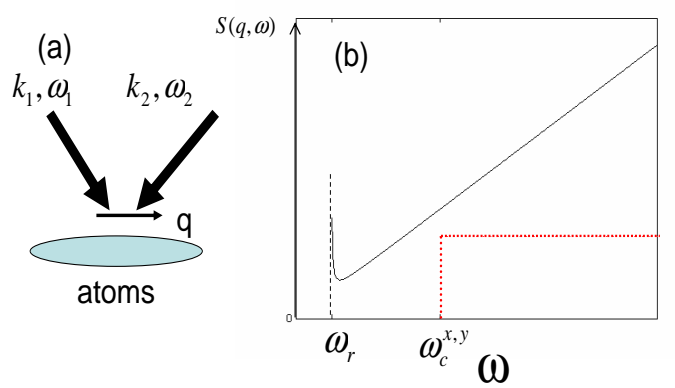

Fig. 8 Schematic of the Bragg scattering. (a) Atoms are illuminated by two laser beams with wave vectors $\mathbf{k}_{1}, \mathbf{k}_{2}$ and frequencies $\omega_{1}, \omega_{2}$, respectively. (b) The dynamic structure factors $S(q, \omega)$ (not scaled) for the massless Dirac fermions (solid line) and for the massive ones in the non-relativistic limit (dotted line). The experimentally measurable quantities $\omega_{r}$ and $\omega_{c}^{x, y}$ give important parameters for the quasiparticles. Reproduced from Ref. [41], Copyright (C) 2007 the American Physical Society.

\section{Quantum anomalous Hall effects}

As a unique electronic behavior of single layer graphene, the relativistic Dirac fermions carrying charge in this 2D material may result in novel transport phenomena. One of the most exotic examples is that, when subjected to a perpendicular magnetic field, the so-call half-integer quantum Hall effect (QHE) as a result of the unconventional relativistic Landau level can be observed in graphene [35,36]. For cold atomic systems, such halfinteger QHE is principally able to be simulated since the relativistic particles emerge [56,61], and the additional requirement is an effective uniform magnetic field, which can be generated by rotation or optical dressing (will be discussed in Section III.A).

In contrast to the conventional QHE and the halfinteger QHE, there is another kind of QHE, called as quantum anomalous Hall effect (QAHE) because the net magnetic flux is zero in each unit cell and there are no Landau levels. However, all the three need the breaking of time-reversal symmetry. Twenty years ago, Haldane proposed a toy model to illustrate such QAHE as a result of the parity anomaly of the 2D Dirac fermions in a hexagonal lattice [81]. Nevertheless, it is extremely hard to realize the Haldane's model experimentally in ordinary condensed matter systems including the graphene because of the unusual staggered magnetic flux assumed in the model. Other schemes have been proposed to realize QAHE in semiconductor systems with topological band structures [82-84], where the time-reversal symmetry is broken by the spin polarization. Besides, it is possible to realize QAHE in graphene in the presence of both Rashba spin-orbit coupling and an exchange field [85], or in a nanopatterned 2D electron gas [86], or in a thin film of a three-dimensional topological insulator with an exchange field [87]. The QAHE states are promising for future device applications because of its potential realization of dissipationless charge transport.
Interestingly, several proposals have been suggested to realize and detect the QAHE by using ultracold atoms. An ingenious scheme is proposed to realize the Haldane' model by using cold atoms trapped in a hexagonal OL with an artificial staggered magnetic field (Berry curvature) with hexagonal symmetry in Ref. [88]. The wanted external field can be achieved by coupling atomic internal state with the standing waves of laser beams. While it remains a challenge to experimentally realize such a staggered magnetic field. In Ref. [89], it was showed that the QAHE can be simulated with the $p$-orbital band in the hexagonal OL through orbital angular momentum polarization by applying a technique of rotating each lattice site around its own center [90]. An expanded version of this proposal can be found in Ref. [91]. Besides, it was recently demonstrated to realize the QAHE with cold atoms trapped in an anisotropic square OL which can be generated from available experimental setups of doublewell lattices with minor modifications [92]. The detection of the QAHE in cold atomic systems can be achieved by direct detection of the Chern number with the typical density-profile-measurement technique [88], or alternatively determining the topological phase transition from usual insulating phase to quantum anomalous Hall phase by light Bragg scattering of the edge and bulk states [92]. All of these proposals involve experimental techniques of implementing cold atoms to be developed.

\section{SIMULATION OF DIRAC EQUATION WITH ULTRACOLD ATOMS IN SYNTHETIC NON-ABELIAN GAUGE FILEDS}

In the models discussed in Sec. II, the emergency of relativistic Dirac fermions (equation) in OL systems generally requires the long wavelength approximation, which supports the continuous limitation. In this section, we introduce another kind of proposals to simulate the tunable Dirac equation that are based on generating effective gauge fields [93-101] on bulk atomic gases, which do not need OLs and thus operates in a continuous regime [102105]. We will see that the effective relativistic dynamics emerge again in the cold atom systems by using atomlight interaction to generate effective gauge potentials acting on these neutral atoms. Besides, there is no filling requirement in these continuous systems, and thus the relativistic Dirac dynamics are equivalent for both noninteracting bosonic and fermionic in the single-particle physics. Moreover, it even holds for the condensation of bosons, which provides us a macroscopic counterpart of relativistic particles described by the nonlinear Dirac equation (NLDE). We first introduce the schemes and experiment progress on creating synthetic gauge potentials in neutral atoms. Then we use two specific systems to show that a liner and nonlinear Dirac equation with tunable parameters through laser fields can be simulated. 


\section{A. Synthetic gauge fields for neutral atoms}

We first briefly review the generation of synthetic gauge fields for neutral cold atoms loaded in OLs. In Section II.A.3, we have mentioned that a U(1) Abelian gauge potential can be generated by atom-laser interaction [52] or temporal modulation of the lattice potential $[53,54]$ for atoms trapped in a square OL. This external gauge potential behaves as a vertical staggered or uniform (when $\gamma$ is spatially independent) magnetic field acting on a charged particle moving in a square potential, and thus contributes a scalar Peierls phase factor to the atomic hopping process. A more straightforward way to generate an Abelian gauge potential in OLs can be achieved by using laser-assisted tunneling for atoms trapped in state-dependent lattice $[71,73]$, where atoms also acquire a phase when tunneling between the two neighbor sublattices. The phase factor can extend to a $2 \times 2$ matrix, corresponding to a square lattice subjected to a U(2) nonAbelian gauge potential [94]. The basic idea to create a $\mathrm{U}(\mathrm{N})$ non-Abelian gauge potential in an OL is to additionally consider atomic species with $N$ internal quasidegenerate sub-levels in both two sublattices [72], and thus instead of a simple phase, the laser-assisted tunneling would induced a rotation in the internal space, described by a $N \times N$ matrix $\mathbf{F}$ with non-commuting component $F_{x}, F_{y}, F_{z}$.

An alternative method to create synthetic gauge field is rotating the neutral atomic gas trapped in harmonic potentials or OLs [106]. This scheme is limited to rotationally symmetric configurations, and furthermore, can not extend to non-Abelian case without additional laser fields [107]. Other schemes that can be used to create a gauge field include using light beams with orbital angular momentum [93-95] or spatially shift laser beams [96-98] to interact with atomic gases. Two reviews on the synthetic gauge potentials for neutral atoms are given in Refs. [99] and [108].

The relativistic Dirac Hamiltonian always includes a spin-orbit coupling term (as shown in Eq. (5)), which can be induced from a U(2) non-Abelian gauge potential. To achieve this goal without OLs, we can consider the scheme based on the adiabatic motion of multiple-level atoms in laser fields that generate (near-)degenerate dark states [100]. This idea goes back to the work by Wilczek and Zee [101], who have shown that non-Abelian gauge fields can arise in the adiabatic evolution of quantum systems with multiple degenerate eigenstates. We consider the adiabatic motion of atoms with $N+1$ internal levels in stationary laser fields, as shown in Fig. 9(a). For fixed position $\mathbf{r}$ the internal Hamiltonian including the laser-atom interaction can be diagonalized to yield a set of $N+1$ dressed states $\left|\chi_{n}(\mathbf{r})\right\rangle$ with eigenvalues $\varepsilon_{n}(\mathbf{r})$, where $n=1,2, \ldots, N+1$. The full quantum state of the atom describing both internal and motional degrees of freedom can then be expanded in terms of the dressed states according to $|\Phi\rangle=\sum_{n=1}^{N+1} \Psi_{n}(\mathbf{r})\left|\chi_{n}(\mathbf{r})\right\rangle$, where the wavefunctions $\Psi=\left(\Psi_{1}, \Psi_{2}, \ldots, \Psi_{N+1}\right)^{\top}$ obeys the
Schrödinger equation

$$
i \hbar \frac{\partial}{\partial t} \Psi=\left[\frac{1}{2 m}(\mathbf{P}-\mathbf{A})^{2}+V\right] \Psi
$$

with $V$ being an external trapping potential. Here the vector potential $\mathbf{A}$ and scalar potential $V$ are $(N+1) \times$ $(N+1)$ matrices come from the spatial dependence of the atomic dressed states with elements

$$
\begin{aligned}
& \mathbf{A}_{n, m}=i \hbar\left\langle\chi_{n}(\mathbf{r}) \mid \nabla \chi_{m}(\mathbf{r})\right\rangle \\
& V_{n, m}=\varepsilon_{n}(\mathbf{r}) \delta_{n, m}+\left\langle\chi_{n}(\mathbf{r})|V(\mathbf{r})| \chi_{m}(\mathbf{r})\right\rangle .
\end{aligned}
$$

If the off-diagonal elements of the matrices $\mathbf{A}$ and $V$ are much smaller than the difference of the dressed atomic energies, we can safely neglect the off-diagonal contributions. In this regime, atoms in any one of the dressed states adiabatically evolve according to a separate Hamiltonian with a U(1) Abelian gauge potential, like a charged particle moving in an electromagnetic field.

The key point to generate non-Abelian gauge potentials is that some atomic dressed states, say the first $q$ ones, form a degenerate (or nearly degenerate) manifold, leading to the failure of adiabatic approximation since some off-diagonal coupling can no longer be ignored. Suppose that these levels are well separated from the remaining ones, the full Hamiltonian can be projected to this subspace for the reduced column vector $\widetilde{\Psi}=\left(\Psi_{1}, \ldots, \Psi_{q}\right)^{\top}$, which obeys the Schrödinger equation $i \hbar \frac{\partial}{\partial t} \tilde{\Psi}=\tilde{H} \tilde{\Psi}$ with the Hamiltonian

$$
\tilde{H}=\frac{1}{2 m}(\mathbf{P}-\mathbf{A})^{2}+V+\Phi
$$

where $\mathbf{A}$ and $V$ being the truncated $q \times q$ matrices with elements defined in Eq. (29), and a scalar potential $\Phi$ arises from the projection, with elements given by

$$
\Phi_{n, m}=\frac{1}{2 m} \sum_{l=q+1}^{N} \mathbf{A}_{n, l} \cdot \mathbf{A}_{l, m} .
$$

Here the effective $\mathrm{U}(\mathrm{q})$ non-Abelian gauge potential A also called the Mead-Berry connection is related to an effective magnetic field (or curvature) $\mathbf{B}$ :

$$
\mathbf{B}=\nabla \times \mathbf{A}+\frac{1}{i \hbar} \mathbf{A} \times \mathbf{A}
$$

Because the vector components of $\mathbf{A}$ do not necessarily commute, the term $\mathbf{A} \times \mathbf{A}$ does not vanish in general, even if the vector potential is uniform in space. In fact, this term reflects the non-Abelian character of the gauge potentials and appears only if $q \geq 2$. When $q=2$ corresponding to a tripod scheme discussed in the following section, under certain conditions the non-Abelian gauge structure is equivalent to a spin-orbit interaction. 
(a)
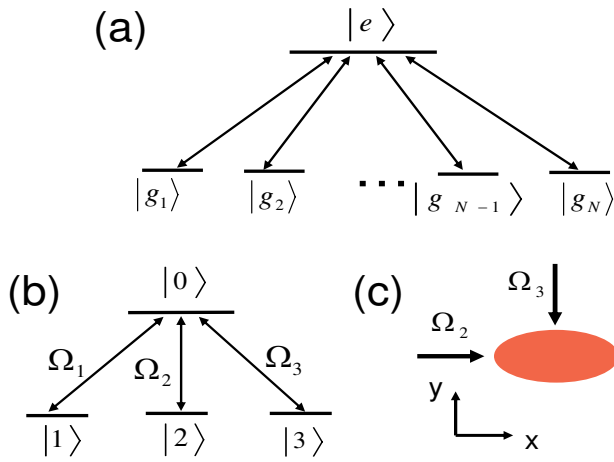

(c)

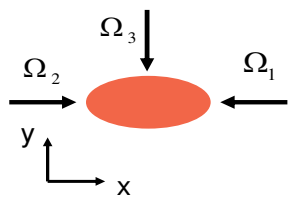

(d)

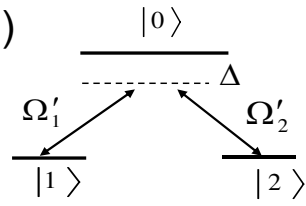

(e)

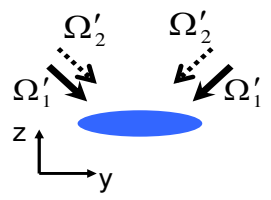

Fig. 9 Schematic of atom-laser interaction. (a) Multi-pod configuration. An atomic state $|e\rangle$ is coupled to $N$ different atomic states $\left|g_{j}\right\rangle(j=1, \ldots, N)$ by $N$ resonant laser fields. (b),(c) Atoms with tripod-level configuration $(N=3)$ interacting with three laser beams characterized by the Rabi frequencies $\Omega_{1}, \Omega_{2}$ and $\Omega_{3}$. (d),(e) Atoms with $\Lambda$-level configuration interacting with laser beams characterized by the Rabi frequencies $\Omega_{1}^{\prime}, \Omega_{2}^{\prime}$ and a large detuning $\Delta$.

The above single particle physics of generating gauge fields is equivalent for bosonic and fermionic atoms. For a many-body system of bosons, such as a BEC, the U(1) Abelian gauge potential acts like a magnetic field and leads to a vortex lattice in the BEC $[98,109]$. The NIST group has recently realized the Abelian gauge potential in a BEC of ${ }^{87} R b$ atoms $[110,111]$. In their experiments, they used a gradient of the detuning of the laser frequency for the Raman transitions between different atomic ground sub-levels to generate an artificial gauge potential, somewhat different from the scheme we discussed above. Besides, the NIST group has simulate an electric force acting on neutral atoms through the time dependence of an effective vector potential [112]. A many-body system of pseudo-spin-1/2 bosons within the tripod scheme was first considered in Ref. [113], and the degenerate condensation ground state of the system was found in the presence of weak interactions. Furthermore, in Refs. [114,115], a spin-orbit coupled spinor BEC was investigated and found to have two different phases in the ground state depending on the interatomic interactions, a single plane wave phase with a single dressed state and a standing wave with a nontrivial spin stripe. Most excitingly, the NIST group in a very recent experiment has reported the first realization of an effective $\mathrm{U}(2)$ non-Abelian gauge potential in neutral atoms - a spin-orbit coupled BEC [116].

\section{B. Tunable Dirac-type equation for cold atoms with tripod- and $\Lambda$ - level structure}

Specializing the above scheme of generating $\mathrm{U}(\mathrm{N})$ nonAblelian gauge potential in neutral atoms, we show in

this section two different systems, where a synthetic $\mathrm{U}(2)$ non-Abelian gauge field appears, can use to simulate the relativistic Dirac equation.

Tripod-level configuration. - Let us first consider the adiabatic motion of atoms in $x-y$ plane with each having a tripod-level structure in a laser field as shown in Fig. 9(b) and (c) $[100,102]$. The atoms in three lower levels $|1\rangle,|2\rangle$ and $|3\rangle$ are coupled with an excited level $|0\rangle$ through three laser beams characterized by the Rabi frequencies $\Omega_{1}=\Omega \sin \theta \mathrm{e}^{-i \kappa x} / \sqrt{2}$, $\Omega_{2}=\Omega \sin \theta \mathrm{e}^{i \kappa x} / \sqrt{2}$ and $\Omega_{3}=\Omega \cos \theta \mathrm{e}^{-i \kappa y}$, respectively, where $\Omega=\sqrt{\left|\Omega_{1}\right|^{2}+\left|\Omega_{2}\right|^{2}+\left|\Omega_{3}\right|^{2}}$ is the total Rabi frequency, and the mixing angle $\theta$ defines the relative intensity. The Hamiltonian of a single atom reads

$$
\hat{H}=\frac{\mathbf{P}^{2}}{2 m}+V(\mathbf{r})+H_{\text {int }},
$$

where $V=\sum_{j=1}^{3} V_{j}(\mathbf{r})|j\rangle\langle j|$ is the external trapping potential and the laser-atom interaction Hamiltonian $H_{\text {int }}$ in the interaction representation is

$$
H_{\text {int }}=-\hbar\left(\Omega_{1}|0\rangle\left\langle 1\left|+\Omega_{2}\right| 0\right\rangle\left\langle 2\left|+\Omega_{3}\right| 0\right\rangle\langle 3|\right)+\text { H.c. }
$$

Diagonalizing the Hamiltonian $H_{\text {int }}$ yields two degenerate dark states of zero energy

$$
\begin{aligned}
& \left|D_{1}\right\rangle=\frac{1}{\sqrt{2}} e^{-i \kappa y}\left(e^{i \kappa x}|1\rangle-e^{-i \kappa x}|2\rangle\right) \\
& \left|D_{2}\right\rangle=\frac{1}{\sqrt{2}} e^{-i \kappa y} \cos \theta\left(e^{i \kappa x}|1\rangle+e^{-i \kappa x}|2\rangle\right)-\sin \theta|3\rangle,(35
\end{aligned}
$$

as well as two bright state separated from the dark states by the energies $\pm \hbar \Omega$. If $\Omega$ is sufficiently large compared to the two-photon detuning due to the laser mismatch and/or Doppler shift, then one can safely study only the internal state of an atom adiabatically evolves within the dark state manifold. In such a resonant coupling configuration, there is atomic dissipation due to the spontaneous emission of the excite state, thus the dark states acquire a finite lifetime. However, it can be up to a few seconds for cold atoms with typical velocity being of the order of a centimeter per second. The lifetime of the dark states can be further increased by using off-resonant coupling configuration as described below.

In the dark state manifold, the atomic state vector $|\Phi\rangle$ can be expanded in terms of the dark states according to $|\Phi\rangle=\sum_{j=1}^{2} \Psi_{j}(\mathbf{r})\left|D_{j}(\mathbf{r})\right\rangle$, where $\Psi_{j}(\mathbf{r})$ is the wave-function for the center-of- mass motion of the atom in the $j$-th dark state. Thus the dynamics of the atom is described by a two-component wavefunction $\tilde{\Psi}=\left(\Psi_{1}, \Psi_{2}\right)^{T}$, which obeys the Schrödinger equation (30) with $q=2$. The non-Abelian gauge potential $\mathbf{A}$ in the present configuration of the light field is of $\mathrm{U}(2)$ nonAbelian one. Substituting Eq. (35) into Eq. (29) and (31), one can work out the expression of potentials $\mathbf{A}, \Phi$ and $V$ :

$$
\mathbf{A}=\hbar \kappa\left(\begin{array}{cc}
\mathbf{e}_{y} & -\mathbf{e}_{x} \cos \theta \\
-\mathbf{e}_{x} \cos \theta & \mathbf{e}_{y} \cos ^{2} \theta
\end{array}\right)
$$




$$
\begin{aligned}
& \Phi=\left(\begin{array}{cc}
\hbar^{2} \kappa^{2} \sin ^{2} \theta / 2 m & 0 \\
0 & \hbar^{2} \kappa^{2} \sin ^{2}(2 \theta) / 8 m
\end{array}\right), \\
& V=\left(\begin{array}{cc}
V_{1} & 0 \\
0 & V_{1} \cos ^{2} \theta+V_{3} \sin ^{2} \theta
\end{array}\right),
\end{aligned}
$$

where the trapping potential is assumed to be the same for the first two atomic states, $V_{1}=V_{2}$. Choose $V_{1}-V_{3}=$ $\hbar^{2} \kappa^{2} \sin ^{2} \theta / 2 m$, and thus the overall trapping potential simplifies to $V+\Phi=V_{1}$ I with I being the unit matrix. Furthermore, let the mixing angle $\theta=\theta_{0}$ to be such that $\sin ^{2} \theta_{0}=2 \cos \theta_{0}$, giving $\cos \theta_{0}=\sqrt{2}-1$. Thus the vector potential $\mathbf{A}$ takes a symmetric form

$$
\mathbf{A}=\hbar \kappa^{\prime}\left(-\mathbf{e}_{x} \sigma_{x}+\mathbf{e}_{y} \sigma_{z}\right)+\hbar \kappa_{0} \mathbf{e}_{y} \mathrm{I}
$$

in terms of the Pauli matrices $\sigma_{x}$ and $\sigma_{z}$, where $\kappa^{\prime}=$ $\kappa \cos \theta_{0}$ and $\kappa_{0}=\kappa\left(1-\cos \theta_{0}\right)$. Using a unitary transformation $\tilde{H}^{\prime}=U^{\dagger} \tilde{H} U$ in Eq. (30) with $U=$ $\exp \left(-i \kappa_{0} y\right) \exp \left(-i \frac{\pi}{4} \sigma_{x}\right)$, one obtains the Hamiltonian for the atomic motion

$$
\tilde{H}^{\prime}=\frac{1}{2 m}\left(\mathbf{P}+\hbar \kappa^{\prime} \sigma_{\perp}\right)^{2}+V_{1},
$$

where $\sigma_{\perp}=\mathbf{e}_{x} \sigma_{x}+\mathbf{e}_{y} \sigma_{y}$ is the spin $1 / 2$ operator in the $x y$ plane. In the limit of low momenta, i.e., $|\mathbf{P}| \ll \hbar \kappa^{\prime}$, one can safely neglect the kinetic energy term in Eq. (38) and obtain an effective Dirac Hamiltonian

$$
H_{2 D}=\hbar c_{0} \mathbf{k} \cdot \sigma_{\perp}+V_{1}+m c_{0}^{2},
$$

where $c_{0}=\hbar \kappa^{\prime} / m$ is the effective light velocity for such quasi-relativistic particles. In this case, the atoms in the system behave as 2D massless relativistic particles.

If we focus on the case with an extremely anisotropic potential, which is sufficiently strong along the transverse direction of the $x$ axis, such that the original massive atoms are actually confined in a one-dimensional guide along the $x$ axis with the vector potential $A_{x}=$ $-\hbar \kappa \cos \theta \sigma_{x}$. In this case, we get a $1 \mathrm{D}$ Dirac equation described by $[103,104]$

$$
H_{1 D}=c_{\star} \sigma_{x} p_{x}+\gamma_{z} \sigma_{z},
$$

up to an irrelevant constant, provided that the wave vector of the atoms $p_{x} / \hbar \ll \kappa \cos \theta$. Comparing the original Dirac equation, here $\gamma_{z} \equiv \hbar^{2} \kappa^{2} \sin ^{4} \theta / 2 m$ is the effective rest energy and $c_{\star}=\hbar \kappa \cos \theta / m$ is the effective light velocity, and thus the effective mass $m_{\star}=\frac{m}{2} \tan ^{2} \theta \sin ^{2} \theta$. Furthermore, if we take $V_{1}-V_{3}=\hbar^{2} \kappa^{2} \sin ^{2} \theta / 2 m$ as discussed above, we are able to obtain a massless 1D Dirac equation when $\gamma_{z}$ in Eq. (40) vanishes. Note that the mass $m_{\star}$ of the simulated Dirac particle is not the mass $m$ of the cold atom itself and it is a remarkable feature that the mass term in the simulated Dirac-like equation can be controlled by the laser beams, so one can simulate a tunable Dirac equation with cold atoms.

$\Lambda$-level configuration. - We now turn to consider the adiabatic motion of atoms in $y$ - $z$ plane with each having a $\Lambda$-level structure interacting with laser beams as shown in Fig. 9(d) and (e) $[105,117]$. The ground states $|1\rangle$ and $|2\rangle$ are coupled to the excited state $|3\rangle$ through laser beams characterized respectively with the Rabi frequencies $\Omega_{1}^{\prime}=\Omega^{\prime} \cos \left(\kappa_{y} y\right) e^{-i \kappa_{z} z}$ and $\Omega_{2}^{\prime}=$ $\Omega^{\prime} \sin \left(\kappa_{y} y\right) e^{i\left(\pi-\kappa_{z} z\right)}$, where $\Omega^{\prime}=\sqrt{\left|\Omega_{1}^{\prime}\right|^{2}+\left|\Omega_{2}^{\prime}\right|^{2}}$. Such Rabi frequencies can be realized with two pairs of planewave lasers as shown in Fig. 9(e). For instance, $\Omega_{1}^{\prime}$ can be realized with a pair of lasers with $\frac{1}{2} \Omega^{\prime} \exp \left[i\left(-\kappa_{z} z \pm \kappa_{y} y\right)\right]$, while $\Omega_{2}^{\prime}$ is achieved with $\frac{1}{2} \Omega^{\prime} \exp \left[i\left( \pm \pi / 2-\kappa_{z} z \pm \kappa_{y} y\right)\right]$, where $\kappa_{y}=\kappa \cos \varphi$ and $\kappa_{z}=\kappa \sin \varphi$ with $\kappa$ being the wave number of the laser beams and $\varphi$ being the angle between the laser beams with the $y$ axis. Such laser beams means that $\Omega_{1}^{\prime}$ and $\Omega_{2}^{\prime}$ are standing waves in the $y$ direction but plane waves in the $z$ direction, and both propagate opposite to the $z$ direction but the phase of $\Omega_{1}^{\prime}$ is $\pi$ ahead. The interaction Hamiltonian in Eq. (33) in this configuration is given by

$$
H_{i n t}^{\prime}=\hbar \Delta|3\rangle\langle 3|-\left(\sum_{j=1}^{2} \hbar \Omega_{j}^{\prime}|3\rangle\langle j|+h . c .\right),
$$

where $\Delta$ is the detuning. Diagonalizing $H_{\text {int }}^{\prime}$ yields a dark eigenstate $\left|\chi^{D}\right\rangle=\sin \left(\kappa_{y} y\right)|1\rangle+$ $\cos \left(\kappa_{y} y\right)|2\rangle$ and two bright eigenstates $\left|\chi_{1}^{B}\right\rangle=$ $(\sin \alpha) \cos \left(\kappa_{y} y\right)|1\rangle-(\sin \alpha) \sin \left(\kappa_{y} y\right)|2\rangle-\cos \alpha e^{-i \kappa_{z} z}|3\rangle$, $\left|\chi_{2}^{B}\right\rangle=(\cos \alpha) \cos \left(\kappa_{y} y\right)|1\rangle-(\cos \alpha) \sin \left(\kappa_{y} y\right)|2\rangle+$ $(\sin \alpha) e^{-i \kappa_{z} z}|3\rangle$, where $\alpha$ is determined by $\tan \alpha=$ $\left(\sqrt{\Delta^{2}+\Omega^{\prime 2}}-\Delta\right) / \Omega^{\prime}$. The corresponding eigenvalues are $E^{D}=0$ and $E_{1,2}^{B}=\hbar\left[\Delta \pm \sqrt{\Delta^{2}+4 \Omega^{\prime 2}}\right] / 2$, respectively. In the large detuning case $|\Delta| \gg \Omega^{\prime}$, the two lower eigenstates $\left|\chi^{D}\right\rangle$ and $\left|\chi_{2}^{B}\right\rangle$ span a near-degenerate subspace, and have negligible contribution from the state of $E_{1}^{B}$. Thus one can safely consider the adiabatic motion of atoms in the near-degenerate subspace, leading to a $\mathrm{U}(2)$ non-Abelian gauge potential with the vector and scalar potentials obtained as $\mathbf{A}=-\frac{\hbar \kappa_{z} \Omega^{\prime 2}}{2 \Delta^{2}} \sigma_{z} \vec{e}_{z}-\hbar \kappa_{y} \sigma_{y} \vec{e}_{y}+$ $\frac{\hbar \kappa_{z} \Omega^{\prime 2}}{2 \Delta^{2}} \mathbf{I} \vec{e}_{z}$ and $\Phi=\frac{\hbar^{2} \Omega^{\prime 2} \kappa^{2}}{4 m \Delta^{2}}\left[\cos (2 \varphi) \sigma_{z}+\mathbf{I}\right]$ in this neardegenerate subspace with the basis $\left\{\left|\chi^{D}\right\rangle,\left|\chi_{2}^{B}\right\rangle\right\}$. Under this condition, we obtain an effective Hamiltonian

$$
\tilde{H}_{k}=\frac{p_{y}^{2}+p_{z}^{2}}{2 m}+v_{y}^{\prime} \sigma_{y} p_{y}+v_{z}^{\prime} \sigma_{z} p_{z}+\gamma_{z}^{\prime} \sigma_{z}+V_{T},
$$

where the parameters $v_{y}^{\prime}=\frac{\hbar \kappa_{y}}{m}, v_{z}^{\prime}=\frac{\hbar \kappa_{z} \Omega^{\prime 2}}{2 m \Delta^{2}}$, and $\gamma_{z}^{\prime}=\frac{\hbar^{2} \Omega^{\prime 2}}{4 m \Delta^{2}}\left[\kappa_{y}^{2}-\left(1+\Omega^{\prime 2} / \Delta^{2}\right) \kappa_{z}^{2}\right]+\frac{\hbar \Omega^{\prime 2}}{2 \Delta}+V_{T}$. In the derivation, one has dropped an irrelevant constant and assumed that the trapping potentials $V_{1,2,3}=V_{T}$ are state-independent. The term related to $\sigma_{z} p_{z}$ in Eq. (42) can be dropped approximately due to $v_{y}^{\prime} \gg v_{z}^{\prime}$. In fact, the dynamics of atoms in the $z$ axis is still governed by the Schrödinger Hamiltonian since the kinetic energy term dominates, and thus the atoms can be well confined by an optical waveguide along $y$ axis [103]. In the limit of low momenta, i.e., $p_{y} \ll \hbar \kappa_{y}$, one can neglect the kinetic energy term. To this step, one obtains the $1 \mathrm{D}$ effective Hamiltonian

$$
H_{1 D}^{\prime}=v_{y}^{\prime} \sigma_{y} p_{y}+\gamma_{z}^{\prime} \sigma_{z}+V_{T},
$$


where $v_{y}^{\prime}$ is the effective speed of light in cold atoms and $\gamma_{z}^{\prime}$ is the effective rest energy, both of which are also controllable by the laser fields.

In the end of this section, we note that the relativistic quasiparticles with total pseudospin $S=1$, which have been described in Section II.A.2, can also be simulated with the tetrapod $(N=4$ in Fig. $9(\mathrm{a}))$ scheme [118]. However, it looks like that the present multi-pod coupling scheme is not feasible to simulate the relativistic quasiparticles with higher (than 1) or even arbitrary pseudospin [118]. To overcome this limitation, one may design other atom-light interaction schemes which remain further work, or one can adopt the proposal described in Ref. [119], where the massless Dirac fermions with arbitrary spin can be simulated by using multicomponent ultracold atoms in a two-dimensional square OL.

\section{Simulation of nonlinear Dirac equation}

In the previous section, we have shown that neutral cold atoms in a light-induced non-Abelian gauge filed can be used to simulate the $2 \mathrm{D}$ and $1 \mathrm{D}$ relativistic Dirac equation with tunable parameters. Note that the above discussion is the single particle physics and is suitable for both noninteracting bosons and fermions. However, atomic interactions are natural and crucial in the atomic assemble, such as BECs. Besides, the artificial nonAbleian gauge potential has been realized in BECs [116]. Thus one may ask whether the relativistic quasiparticles can emergence in a many-body cold atom system. In this section, we shall address that in the presence of a laserinduced spin-orbit coupling an interacting ultracold BEC may acquire a quasi-relativistic character described by a nonlinear Dirac-like equation [105,120].

In the tripod scheme [120], one may choose another three laser beams to get a more symmetrical gauge potential $\mathbf{A}=-\hbar \kappa \sigma_{y} \vec{e}_{x}$. This may be achieved by employing three co-propagating lasers along the $\mathrm{z}$ axis, with constant $\left|\Omega_{3}\right|$ and spatially dependent transversal profiles $\Omega_{1}=\left|\Omega_{3}\right| \cos \phi(x, y) e^{i \kappa z}, \Omega_{2}=\left|\Omega_{3}\right| \sin \phi(x, y) e^{i \kappa z}$ with a laser density modulation along $x$ such that $\phi(\mathbf{r})=$ $\sqrt{2} \kappa x$. If the density modulation has however a polar symmetry on the $x y$ plane, i.e., $\phi(\mathbf{r})=\sqrt{2} \kappa \rho$ (with $\rho^{2}=x^{2}+y^{2}$ ), then $\mathbf{A}=-\hbar \kappa \sigma_{y} \vec{e}_{\rho}$, which is the $2 \mathrm{D}$ case. Finally, setting state-dependent trapping potential $V_{1}=V_{2}=\hbar \Delta_{T}-\hbar^{2} \kappa^{2} / 2 m$ with a detuning $\Delta_{T}$, and $V_{3}=-V_{1}-2 \hbar \Delta_{T}$, one obtains for both $1 \mathrm{D}$ and $2 \mathrm{D}$ arrangements $\boldsymbol{\Phi}+V=\hbar \Delta_{T} \sigma_{z}$.

Interactions are characterized by $s$-wave scattering, and the scattering lengths of different internal states are in principle different, which may present collisionally induced spin rotations. For simplicity of the discussion, we consider that the interaction among the particles of different internal states are all identical, with an effective $d$-dimensional interacting strength $g=$ $4 \pi \hbar^{2} a_{s} N /\left(m \sqrt{2 \pi} l_{\perp}^{2}\right)^{3-d}$, where $a_{s}$ is scattering length, $N$ is the particle number, and $l_{\perp}$ is the oscillator length associated to a harmonic vertical confinement. We also assume that the interaction energy is much smaller than $\hbar \Omega$, such that we remain in degenerate subspace. Considering a wave-packet with $\langle p\rangle=\hbar k_{0} \ll \hbar \kappa$ and momentum width $\delta p \ll 2 \hbar \kappa$, we can still safely neglect the $\mathbf{p}^{2}$ term. Within the Gross-Pitaevskii formalism, the interacting bosons in the degenerate subspace manifold are then effectively described by a NLDE as $i \hbar \partial_{t} \Psi=H_{N D} \Psi$, where

$$
H_{N D}=\frac{\hbar \kappa}{m} \mathbf{p} \cdot \sigma+\hbar \Delta_{T} \sigma_{z}+g \Psi^{\dagger} \cdot \Psi .
$$

Here the spin-orbit coupling term $\mathbf{p} \cdot \sigma=p_{x} \sigma_{y}$ for the $1 \mathrm{D}$ case, and for the $2 \mathrm{D}$ case $\mathbf{p} \cdot \sigma=p \sigma_{y}$ with $p^{2}=p_{x}^{2}+p_{y}^{2}$.

For the $\Lambda$-level scheme [105], the requirements of weak atomic interactions and small momentum width for obtaining the NLDE are similar with those in tripod scheme, i.e., the interaction energy is much smaller than $\hbar \Omega^{\prime}$ and $\delta p_{y} \ll 2 \hbar \kappa_{y}$. In this configuration, the dynamics of a spin-orbit coupled BEC are effectively described by the 1D NLDE with the Hamiltonian

$$
H_{N D}^{\prime}=-i \hbar v_{y}^{\prime} \sigma_{y} \partial_{y}+\gamma_{z}^{\prime} \sigma_{z}+g \Psi^{\dagger} \cdot \Psi+V_{T} .
$$

Up to this, we have addressed that the dynamics of a BEC with a light-induced spin-orbit coupling can effectively described by a $1 \mathrm{D}$ or $2 \mathrm{D}$ NLDE under certain conditions. Such quasi-relativistic BECs may become self-trapped and resemble the so-called chiral confinement studied in the context of the massive Thirring model [120]. In Section IV.C, however, we will show that macroscopic relativistic tunneling effect can be detected in the $\Lambda$-scheme system.

Before closing this section, we note that the NLDE may also be simulated with a BEC loading in the hexagonal optical lattice, proposed in Refs. [121-123]. It was suggested that the NLDE can be obtained by cooling bosons to form a condensation in the lowest Bloch band of a hexagonal optical lattice and then adiabatically translate the BEC to the Dirac point at the band edge by adiabatically tuning the relative phases between the laser beams $[121,122]$. In this system, the interplay between nonlinear atomic interactions and Dirac dynamics may lead to rich localized excitations [122], including solitons, vortices, skyrmions, and half-quantum vortices. However, it was recently argued that the tight-binding model to describe such an interacting boson system around the Dirac point is inadequate [123]. To support this point, it was showed that the atomic interaction of arbitrary small strength can completely deform the topological structure of the Bloch bands at the Dirac point [123].

\section{OBSERVATION OF SOME RELATIVISTIC EFFECTS}

Some relativistic effects related to the Dirac equation, have been predicted for a long time, the most well-known ones should be the Zitterbewegung (ZB) effect and the 
Klein tunneling (KT). The concept of ZB was first introduced by Schrödinger [39], who calculated the resulting time dependence of the electron velocity and position in the framework of the Dirac equation. He found that, in addition to classical motion, a free relativistic electron exhibits a rapid periodic oscillations. The $\mathrm{ZB}$ effect is due to an interference between positive and negative energy state [38], and will disappears with time if an electron is represented by a wave packet since the interference has a finite lifetime. Though Schrödinger's idea stimulated numerous theoretical investigation, direct experimental observation seems impossible for free electrons since the predicted frequency $\hbar \omega_{Z} \simeq 2 m_{e} c^{2} \simeq 1 \mathrm{MeV}$ (here $m_{e}$ is the mass of an electron) and the amplitude $\lambda_{c}=h / m_{e} c \simeq 3.86 \times 10^{-3} \AA$.

Another exotic relativistic phenomena in the Dirac equation was pointed out by Klein, who first used the Dirac equation to study an electron scattering by a po tential step and found that there exists a nonzero trans mission probability even though the potential hight tend to infinity [40], in contrast to the scattering of a non relativistic particle. This unique scattering process ha attracted lots of interest over the past eighty years bu failed to be directly tested by elementary particles du to the requirements of currently unavailable electric fiels gradients, such as on the order of $2 m_{e} c^{2} / e \lambda_{c} \sim 10^{18} \mathrm{~V} / \mathrm{n}$ for free electrons.

In this section, we demonstrate that the famous ZI and KT can both be observed in cold atom systems where the dynamics of the quasiparticles are describer justly by the relativistic Dirac equation. It is a remark able feature that the effective mass and the effective ligh speed are both controllable by the laser fields, whic] makes the observation region can be achieved in experiments. In principal, the OL systems discussed in Section II and the bulk atom systems without OLs discussed in Section III, are both able to exhibit the relativistic effects, we however here focus on the latter for consistency but without loss of generality. We are also interested in the tunneling issues of a spin-orbit coupled BEC described by a NLDE, which may support the direct observation of macroscopic $\mathrm{KT}$ under realistic conditions.

\section{A. Zitterbewegung}

The concept of ZB was first rooted in the Dirac equation, however, its present is not unique to the relativistic electrons, but rather than a generic feature of spinor systems with Dirac-type dispersion relationship. In solid state systems, the ZB can be viewed as a special kind of interband transitions with generation of virtual electron-hole pairs [124]. The energy gaps in these systems are usually of the order of $\mathrm{meV}$ and the oscillation frequency is typically a few THz. Particularly, semiconductor quantum wires [125], graphene [126,127], superconductors [128], and photonic crystals $[129,130]$, trapped ions $[10,131]$ have been proposed as candidate systems for the obser- vation of ZB. Excitingly, a proof-of-principle quantum simulation of the ZB has been respectively performed in the recent experiments in a photonic crystal [131] and trapped ion system [10]. Since a Dirac-like Hamiltonian can be created by cold atoms coupling with laser beams, atoms in tripod-scheme system undergo ZB as a result $[104,132]$. It is also possible to realize $\mathrm{ZB}$ with cold atoms in an Abelian vector potential [133], and even to control the amplitude and lifetime of $\mathrm{ZB}$ via an additional mirror oscillation [134]. The ZB can also be simulated with cold atoms in a driven [135] or titled OL [136]. In these cold atomic systems, typically, the frequency of $\mathrm{ZB}$ is on the order of a few $\mathrm{MHz}$ or $\mathrm{kHz}$ and the amplitude is comparable to the wavelength of the laser beams. Thus they are promising candidates for observing ZB. Here we give a complementary example to show the atomic $\mathrm{ZB}$ by using the $\Lambda$ - scheme.
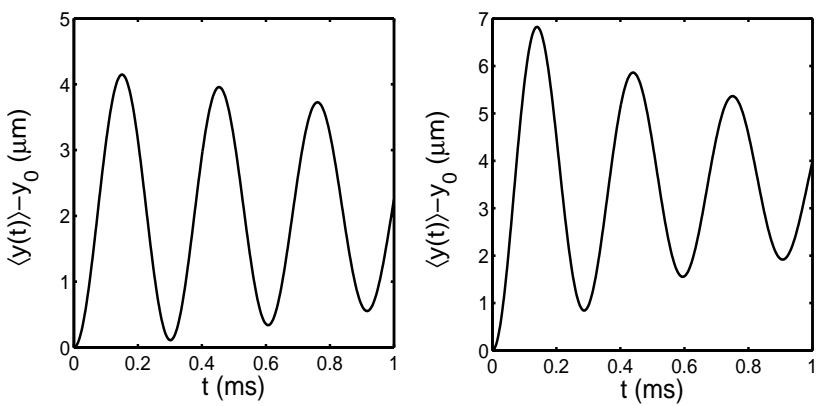

Fig. 10 Zitterbewegung for wave packets of ${ }^{7} \mathrm{Li}$ atomic gas with initial wave number $k_{0}=0$. (a) $\kappa_{y}=5.0 \times 10^{6} \mathrm{~m}^{-1}$, (b) $\kappa_{y}=$ $1.0 \times 10^{7} \mathrm{~m}^{-1}$. Other parameters are $m=1.16 \times 10^{-26}, \gamma_{z}^{\prime} / \hbar=10$ $\mathrm{kHz}$ and $\delta_{l}=10 \mu \mathrm{m}$, respectively.

In the $\Lambda$-scheme, the dynamics of atoms are effectively described by the Dirac-like Hamiltonian (44). We consider the Gaussian wave packet as the initial wave function

$$
\Psi(y, 0)=\frac{1}{\sqrt{\delta_{l} \sqrt{\pi}}} e^{i k_{0} y} e^{-\left(y-y_{0}\right)^{2} / 2 \delta_{l}^{2}}\left(\begin{array}{c}
c_{1} \\
c_{2}
\end{array}\right)
$$

in the spatial space, where $\left|c_{i}\right|^{2}(i=1,2)$ normalized as $\left|c_{1}^{2}\right|+\left|c_{2}^{2}\right|=1$ determine the initial population in states $\left|\chi_{i}\right\rangle, \delta_{l}, k_{0}$ and $y_{0}$ are initial wave-packet width, initial averages of wave number and position, respectively. We suppose $\delta_{l}=\sqrt{\hbar / m \omega_{T}}$, corresponding to the groundstate width of the trapping potential $\frac{1}{2} m \omega_{T}^{2} y^{2}$ in $y$-axis. In the momentum space, the initial wave packet is given by

$$
\begin{aligned}
& \Phi\left(k_{y}, 0\right)=\frac{1}{\sqrt{2 \pi}} \int \Psi(y, 0) e^{-i k_{y} y} d y \\
& \quad=\frac{1}{\sqrt{\delta_{k} \sqrt{\pi}}} \times e^{-i\left(k_{y}-k_{0}\right) y_{0}} e^{-\left(k_{y}-k_{0}\right)^{2} / 2 \delta_{k}^{2}}\left(\begin{array}{c}
c_{1} \\
c_{2}
\end{array}\right),
\end{aligned}
$$

where $\delta_{k}=\delta_{l}^{-1}$ is the momentum spread. When $t=0$, one turns off the trapping potential $V_{T}$, and after $t$ time evolution governed by Dirac-type Hamiltonian $H_{1 D}^{\prime}$ with $V_{T}=0$, the finial wave function is written as 


$$
\Psi(y, t)=\hat{\mathcal{T}} e^{-\frac{i}{\hbar}\left(v_{y}^{\prime} \sigma_{y} p_{y}+\gamma_{z}^{\prime} \sigma_{z}\right) t} \Psi(y, 0),
$$

where $\hat{\mathcal{T}}$ denotes the time ordering operator. It is straightforward to show its time evolution $\Psi(y, t)=$ $\frac{1}{\sqrt{2 \pi}} \int \Phi\left(k_{y}, t\right) e^{i k_{y} y} d k_{y}$ with

$$
\begin{aligned}
& \Phi\left(k_{y}, t\right)=\frac{1}{\sqrt{\delta_{k} \sqrt{\pi}}} e^{-i\left(k_{y}-k_{0}\right) y_{0}} e^{-\left(k_{y}-k_{0}\right)^{2} / 2 \delta_{k}^{2}} \\
& \quad \times\left(\begin{array}{c}
c_{1} \cos \left(\omega_{k} t\right)-\frac{v_{y}^{\prime} k_{y} c_{2}+i \gamma_{z}^{\prime} c_{1} / \hbar}{\omega_{k}} \sin \left(\omega_{k} t\right) \\
c_{2} \cos \left(\omega_{k} t\right)+\frac{v_{y}^{\prime} k_{y} c_{1}+i \gamma_{z}^{\prime} c_{2} / \hbar}{\omega_{k}} \sin \left(\omega_{k} t\right)
\end{array}\right),
\end{aligned}
$$

where $\omega_{k}=\sqrt{\left(\gamma_{z}^{\prime} / \hbar\right)^{2}+\left(v_{y}^{\prime} k_{y}\right)^{2}}$ leads to population transfer between two spin states, and thus $\Psi(y, t)$ is sensitive to the initial spinor components. If we choose $c_{1}=c_{2}=1 / \sqrt{2}$, the wave packet of atomic gas undergoes ZB $[104,130]$. To investigate atomic ZB, we calculate the expectation value of the center of mass, which is given by

$$
\begin{aligned}
\langle y(t)\rangle & =i \int d k_{y} \Phi^{\dagger}\left(k_{y}, t\right) \partial_{k_{y}} \Phi\left(k_{y}, t\right) \\
= & y_{0}+\frac{v_{y}^{\prime} \gamma_{z}^{\prime}}{\hbar \delta_{k} \sqrt{\pi}} \int d k_{y} \frac{\sin ^{2}\left(\omega_{k} t\right)}{\omega_{k}^{2}} e^{-\left(k_{y}-k_{0}\right)^{2} / \delta_{k}^{2}} .
\end{aligned}
$$

The integral term stands for ZB which shows the oscillation of the center-of-mass motion. To have some ideas about ZB, we numerically calculate Eq. (50) and two examples are shown in Fig. 10. The reason for $\mathrm{ZB}$ is the separation of initial wave packet by spin and then interfere between the coupling components, which gives rise to oscillation of center-of-mass motion. ZB oscillations for finite momentum spread damp out over time, and its lifetime can be increases by reducing momentum spread $\delta_{k}$. In this model, the amplitude of ZB is primarily determined by the central momentum $k_{0}$ and the spin-orbit coupling strength $v_{y}^{\prime}$. The smaller $k_{0}$ and the larger $v_{y}^{\prime}$, the larger amplitude of ZB. The amplitude of ZB here can reach a few $\mu \mathrm{m}$ and it is around $7 \mu \mathrm{m}$ when $k_{0}=0$, as shown in Fig. 10(b), which is larger than that in early proposals on observations of ZB with cold atoms [104,132-134]. Meanwhile, the atomic ZB can persist for several milliseconds with frequency being of the order of a few $\mathrm{kHz}$. $\mathrm{ZB}$ of atomic gas with such large amplitude and long lifetime is detectable in current experiments.

\section{B. Klein tunneling}

The KT is originally referred to the step potential, however, it has been extended to other kinds of potential barriers, leading to a general description that relativistic particles can penetrate through high and wide potential barriers without exponential damping expected in nonrelativistic tunneling processes. Though this interesting relativistic scattering process can not be directly tested by elementary particles, some quasiparticles in certain systems may be described by effective relativistic wave equation and thus provide a platform to simulate the KT. Those including electrons in graphene [137], electromagnetic waves in honeycomb photonic crystals [138], cold atoms in a driven [135] or bichromatic [139,140] optical lattice, confined stationary light [141], and trap ions [131], have been proposed to observe such relativistic tunneling. Interestingly, in two very recent experiments, a proof-of-principle simulation of KT with trapped ions [11] and with ultracold atoms in a bichromatic optical lattice [140] has been performed, respectively. We in the following review a proposal of directly observing the KT with cold atoms in a non-Abelian gauge field generated by using the $\Lambda$-scheme [105], as discussed in Section III.B, where we have obtained a Dirac-like equation (43) with tunable parameters. (a)

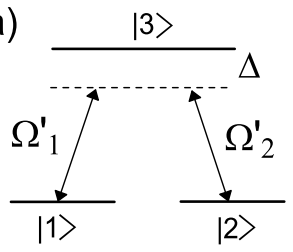

(b)

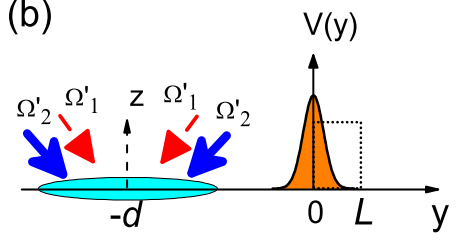

Fig. 11 Schematic illustration of the system. (a) Atom with $\Lambda$-level configuration interacting with laser beams. (b) The configuration of the laser beams to realize a Dirac-like equation and an effective Gaussian (square)-shape potential induced by another laser beam. The atoms are confined in a 1D waveguide along $y$ axis and scattered by the potential.

To have an intuitive physics picture, we first consider a single atom with energy $E$ scattered by a square potential with the width $L$ and potential hight $V_{s}$, as shown in Fig. 11(b). Such an effective square potential can be experimentally formed by a laser beam with flat-top profile [142]. The transmission coefficient $T_{D}$ for the socalled $\mathrm{KT}$ regime is given by

$$
T_{D}=\left[1+\left(\eta-\eta^{-1}\right)^{2} \sin ^{2}(\alpha L) / 4\right]^{-1}
$$

where $\eta=\sqrt{\frac{\left(V_{\mathrm{s}}-E+\gamma_{z}^{\prime}\right)\left(E+\gamma_{z}^{\prime}\right)}{\left(E-V_{\mathrm{s}}+\gamma_{z}^{\prime}\right)\left(\gamma_{z}^{\prime}-E\right)}}$ and $\alpha=\sqrt{\frac{\left(V_{\mathrm{s}}-E-\gamma_{z}^{\prime}\right)}{\left(V_{\mathrm{s}}-E+\gamma_{z}^{\prime}\right)}} / \hbar$. Compared with the familiar property in the textbook of non-relativistic quantum mechanics that the transmission coefficient is a mono exponential decreasing as a function of the width $L$ or the potential height $V_{s}$, a distinguished different feature within this relativistic tunneling region is that the tunneling amplitude can be an oscillation function of $V_{s}$ or $L$ even when the kinetic energy of the incident particle is less than the height of the square barrier potential. This relativistic effect can be attributed to the fact that the incident particle in positive energy state can propagate inside the barrier by occupying a negative energy state, which is also a plane wave aligned in energy with that of the particle continuum outside. Matching between positive and negative energy sates across the barrier leads to the high-probability tunneling. Furthermore, the resonant transmission occurs for perfect matching where the potential width equates integral multiple of half-wavelength of the negative energy state, corresponding to $\alpha L=n \pi(n=1,2, \cdots)$ in 
the formula of $T_{D}$. We take the atoms of ${ }^{7} \mathrm{Li}$ with mass $m=1.16 \times 10^{-26} \mathrm{~kg}$ as an example. If we choose the following typical experimental parameters: the wave numbers $k_{a}=1.1 \times 10^{6} \mathrm{~m}^{-1}, \kappa_{y}=10^{7} \mathrm{~m}^{-1}, \kappa_{z}=0.8 \times 10^{7}$ $\mathrm{m}^{-1}$, the Rabi frequency $\Omega=10^{7} \mathrm{~Hz}$ and the detuning $\Delta=10^{9} \mathrm{~Hz}$, we can find that the Klein regime corresponds to the required potential height $V_{s}>0.162$ $\hbar \times \mathrm{MHz}$, which can be easily achieved in experiments. So we have demonstrated from a simple example that it is feasible to observe the $\mathrm{KT}$ with cold atoms.

As for a practical experiment we are required to release two conditions: the trajectory of a single atom is hard to detect, and it is much easier in experiments to measure the density evolution of an ensemble of noninteracting atoms. Compared with a perfect square potential barriers achieved with a flat-top laser beams, a Gaussian potential barrier $V_{b}^{\mathrm{G}}(y)=V_{\mathrm{G}} e^{-y^{2} / \sigma^{2}}$, where $V_{\mathrm{G}}$ is the height and $\sigma$ characterizes the corresponding spatial variance, is much easier to be generated. However, the conditions of resonant transmission varies with the velocity and the width of the potential, and thus both the ensemble of atoms and the Gaussian potential may smooth the oscillations in the transmission coefficient.

So we now turn to address an ensemble of atoms that are scattered by the Gaussian potential. We assume that the ensemble of atoms are initially trapped in a harmonic trap which moves along the $y$ axis with the wave number $k_{a}$. At the beginning $t=0$, the center of the harmonic trap locates at $y=-d$, and the center of the Gaussian potential barrier is at $y=0$, as shown in Fig. 11. In this case the number density and the number wave number of the atomic ensemble are characterized by Gaussian distribution. The trap is turned off at $t=0$ and then we calculate the evolution of the density profile of the atomic gas after a long enough time for scattering. We define the average transmission coefficient for an ensemble of $N_{a}$ noninteracting atoms as

$$
\langle T\rangle=\frac{1}{N_{a}} \sum_{i=1}^{N_{a}} T\left(k_{i}\right),
$$

where $T\left(k_{i}\right)$ denotes the transmission coefficient of the atom $i$ scattered by the Gaussian potential, as a function of random wave number $k_{i}$ described by the Gaussian distribution $k_{i} \sim N\left(k_{a}, \sigma_{k}^{2}\right)$. The analytical expression of $T\left(k_{i}\right)$ is absent, however, we here show an efficient method to numerically solve it based on the transfer matrix methods. The numerical procedures are outlined as follow. One first cuts the Gaussian potential into spatially finite range $y \in\left[-y_{c}, y_{c}\right]$, where the cutoff position $y_{c}$ should be chosen to guarantee that the potential height outside the range is low enough to be transparent for the atoms, i.e., $V_{b}^{\mathrm{G}}\left(y_{c}\right) \ll E, V_{\mathrm{G}}$. Secondly, one equally divides this range into $n$ spindly segments and each segment can be considered as a square potential if $n$ is large enough. Thus the Gaussian potential can be approximately viewed as a sequence of connective small square potential barriers, and the transmission coefficient
$T\left(k_{i}\right) \approx 1 /\left|m_{11}\right|^{2}$, where $m_{11}$ is the first element of the whole transfer matrix $M=M_{n} M_{n-1} \cdots M_{j} \cdots M_{2} M_{1}$. Here $M_{j}$ denotes the transfer matrix of the $j$-th square potential barrier, whose explicit elements can be found in Ref. [103]. Note that this numerical calculation scheme recovers the non-relativistic scattering governed by the Schrödinger equation.
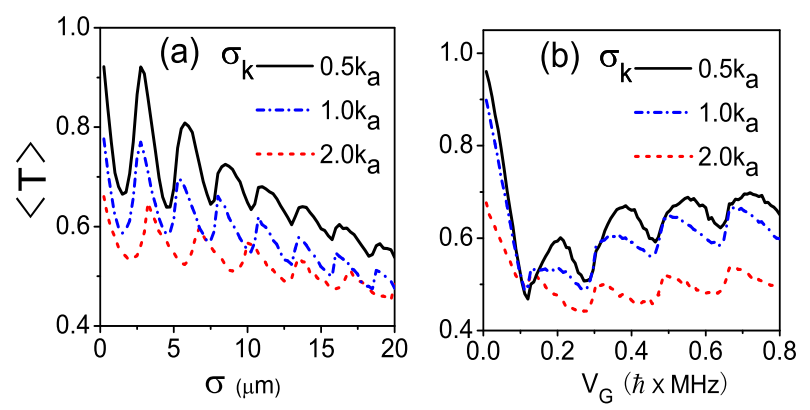

Fig. 12 Klein tunneling of an ensemble of $N_{a}=10^{4}$ noninteracting atoms. (a) $\langle T\rangle$ as a function of potential width $\sigma$ with $\Omega_{b}^{\mathrm{G}}=0.8$ MHz. (b) $\langle T\rangle$ as a function of potential hight $V_{\mathrm{G}}$ with $\sigma=10 \mu \mathrm{m}$. The black solid line, blue dashed line and red dotted line in both (a) and (b) correspond to the cases of $\sigma_{k}=0.5,1.0,2.0 k_{a}$, respectively. Other parameters are $k_{a}=1.1 \times 10^{6} \mathrm{~m}^{-1}, \kappa_{y}=10^{7} \mathrm{~m}^{-1}$, $\kappa_{z}=0.8 \times 10^{7} \mathrm{~m}^{-1}$, frequency $\Omega=10^{7} \mathrm{~Hz}$, and $\Delta=10^{9} \mathrm{~Hz}$.

Figure 12 shows the average transmission coefficient $\langle T\rangle$ of an ensemble of $N_{a}=10^{4}$ noninteracting atoms as a function of the width $\sigma$ and the height $V_{G}$ of the Gaussian potential. Similar with the results for the single atom, the tunneling oscillation still survives after the average of the random distribution of the velocities for the Gaussian potential. The amplitude of oscillation decreases with the increasing of the wave number variance. Such KT features can be attributed to the fact that the tunneling of an ensemble of noninteracting atoms is equivalent to the superposition of every single-atom tunneling with different oscillation period and amplitude, leading to smoothing the oscillation property. The large velocity variance, the more obvious smoothing effects. The oscillation period of single-atom tunneling is insensitive to the potential hight in contract to the potential width, and thus almost no decay of oscillation in Fig. 12(b). In particular the interesting $\mathrm{KT}$ can be observed under realistic conditions. For instance, the difference between the nearest peaks of $\langle T\rangle$ in spatial dimension are in the region $3.0-6.0 \mu \mathrm{m}$, and the frequency is about $0.2 \mathrm{MHz}$. Both of them in the oscillation are detectable within the current technology.

\section{Macroscopic Klein tunneling}

Although it is possible to observe the KT at a relatively high temperature, the phenomena is clearer in low temperature. In low temperature the bosonic atoms may form the BEC. Moreover, the gauge field has recently been generated in a BEC by the NIST group [111,116], thus it deserves to study whether the $\mathrm{KT}$ is still de- 
tectable with a BEC. Surprisingly we will illustrate below that the KT of a spin-orbit coupled BEC may be observed very clearly [105].

The single-atom dispersion is characterized by two branches $E_{ \pm}\left(k_{y}\right)= \pm\left(\gamma_{z}^{\prime 2}+\hbar^{2} v_{y}^{2} k_{y}^{2}\right)^{1 / 2}$, where the lower (up) branch represents the negative (positive) energy state. One can prepare an initial BEC with a designated mode $k_{0}$ at the positive or negative energy branch. The two branches allow us to investigate a more fruitful tunneling problem: there are four classes of the scattering which describe the wave function $\Psi_{\mu}(\mu(= \pm))$ scattered by the potential $\nu V_{b} \mathrm{G}$ with $\nu$ donating a barrier $(\nu=+)$ or a potential well $(\nu=-)$, as shown in Fig. 13(a). We assume that the BEC is initially trapped in a harmonic trap which moves along the $y$ axis, thus we may choose the initial wave function of the BEC as

$$
\Psi_{\mu}(y, 0)=\frac{1}{\sqrt{l_{0} \sqrt{\pi}}} e^{i \mu k_{0} y} e^{-(y+d)^{2} / 2 l_{0}^{2}} \phi_{\mu},
$$

where $l_{0}$ is the width, $k_{0}$ is the central wave number of the wave-packet and the spinor $\phi_{\mu}$ are defined as $\phi_{+}=(i \cos \xi,-\sin \xi)^{T}, \phi_{-}=(-i \sin \xi, \cos \xi)^{T}$ with $\xi=\frac{1}{2} \arctan \left(\hbar v_{y} k_{0} / \gamma_{z}^{\prime}\right)$ and $T$ as the transposition of matrix. Equation (53) describes the Gaussian wave packet with the central velocity $\hbar\left(\kappa_{y}+\mu k_{0}\right) / m$ moving along $y$-axis. After the evolution governed by Dirac-type (45) with time $t$ and replacing $V_{T}$ by $\nu V_{b}^{\mathrm{G}}(y)$, the finial wave function becomes

$$
\Psi_{\mu}(y, t)=\hat{\mathcal{T}} \exp \left(-\frac{i}{\hbar} \int_{0}^{t} H_{N D}^{\prime} d t\right) \Psi_{\mu}(y, 0) .
$$

We have numerically calculated $\Psi_{\mu}(y, t)$ and as an example being shown in Fig. 13(b), the stationary solution of the tunneling is always found within a few milliseconds, which is a small time scale comparing with the lifetime of BECs. After tunneling, the incident wave packet divides into the left- and right-traveling wave packets and only the latter one is on the transmission side of the barrier. Thus we can define the transmission coefficient of the incident wave packet $\Psi_{\mu}(y, 0)$ scattering by a potential $\nu V_{b}{ }^{\mathrm{G}}$ as

$$
T_{\mu \nu}=\int_{\sigma}^{\infty} \Psi_{\mu}^{\dagger}(y, \tau) \Psi_{\mu}(y, \tau) d y
$$

where $\tau$ (being slightly larger than $d / v_{0}$ ) represents a time that the reflected and transmitted wave packets are sufficient away from the Gaussian potential. One can directly measure the transmission coefficient in Eq. (55) since the spatial density distribution $\rho_{\mu}(y, \tau)=\left|\Psi_{\mu}(y, \tau)\right|^{2}$ can be detected using absorption imaging [143]. We first look into the tunneling phenomena for a non-interacting BEC ( $g=0$ in Eq. (45)) realized by Feshbach resonance [143], and then briefly discuss the effects of the interaction between the atoms.

We plot the transmission coefficient $T_{++}$as a function of the potential height $V_{\mathrm{G}}$ and width $\sigma$ in Fig. 14(a,b) with the practical experimental parameters. It is inter- esting to note that the transmission coefficient decreases exponentially to zero with $V_{\mathrm{G}}$ when $V_{\mathrm{G}}<V_{\mathrm{G}}^{K}$, while if we further increase the potential height to the Klein region $V_{\mathrm{G}}>V_{\mathrm{G}}^{K}$, the transmission coefficient will increase and then be an oscillating function in the Klein region $V_{\mathrm{G}}>V_{\mathrm{G}}^{K}$, being similar to the results of Eq. (51) for the which describes the transmission of the square potential barrier. Here the critical value of the potential height may approximately be estimated using the square potential barrier with $V_{\mathrm{G}}^{K}=E\left(k_{0}\right)+\gamma_{z} \approx 0.09 \mathrm{MHz}$. Besides, there are two identities $T_{++}=T_{--}$and $T_{-+}=T_{+-}$since Eq. (45) with $g=0$ is invariant under the charge conjugation [144], the former as an example is confirmed in Fig. 14(a). Although the amplitude of tunneling oscillation is less than the unit as comparing with the tunneling of single atom, the amplitude of tunneling oscillation can be more than 0.5 and meanwhile the period can be a few micrometers as shown in Fig. 14(b), which is experimentally detectable.

The most exotic feature induced by the relativistic effects is that, the BEC with negative energy can almost completely transmit a wide Gaussian potential barrier, as shown in Fig. 14(c). This phenomena can be attributed to the fact that the incident $\mathrm{BEC}$ in a negative energy level can be considered as a macroscopic 'anti-BEC'. Alternatively, it can be understood that such scattering feature is actually equivalent to that of a BEC of positive energy scattered by a Gaussian potential well because of $T_{-+}=T_{+-}$. We also calculate the transmission coefficient for the central mode of the wave packet, as shown in the insert of Fig. 14(c), which further confirms that a wide enough Gaussian potential well is transparent. The reason lies in the fact that, in contract to square potential wells, the Gaussian potential wells are smooth (without any energy jump) in the whole space, and may support adiabatic motions of wave packets in the large width limitation. (a)

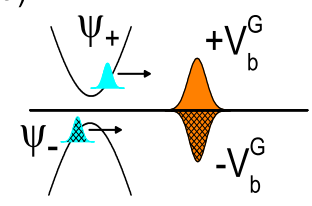

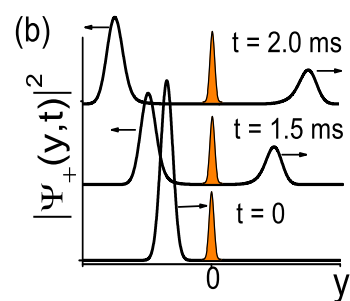

Fig. 13 Schematic representation of the scattering of a BEC. (a) A schematic diagram shows four kinds of scattering events. (b) Normalized density distribution in a scattering process at time $t=0,1.5$ and $2.0 \mathrm{~ms}$. The peaks at $y=0$ are the Gaussian barriers.

The exotic tunneling property exhibited in Fig. 14(c) is an intrinsic relativistic and macroscopic quantum phenomenon that can not be explained with an incoherent ensemble average of a large number of atoms. To clarify this point we also calculate numerically the average transmission coefficient for an ensemble of $10^{4}$ noninteracting atoms as $\langle T\rangle$. Here we choose the wave number distribution to be the same Gaussian distribution as that in Eq. (53), i.e., $k_{i} \sim N\left(k_{0}, \sigma_{k}^{2}\right)$ with the variance $\sigma_{k}=1 / l_{0}$. 
The average coefficient $\langle T\rangle$ is shown in the inset of Fig. 14(c), which is almost the same as that of a single atom since $\sigma_{k}$ is small. The differences between $\langle T\rangle$ and $T_{-+}$in Fig. 14(c) clearly demonstrate that the tunneling of BEC is not equivalent to an ensemble average of the individual atoms with the same distribution of wave number.

Before ending this section, we make two additional comments. (i) The quadratic term of the momentum in Eq. (42) has been neglected in the derivation of the Dirac Eq. (45). To judge the feasibility of this approximation, the transmission coefficients $T_{++}$with or without the quadratic term are compared in Fig. 14(b). It is shown that the quadratic term leads to merely a slight left-shift of the tunneling peaks. This phenomenon can be interpreted by the fact that the wavelength of the 'anti-particle' consisting of the BEC inside the barrier decreases slightly in the presence of the additional low kinetic energy. This result verifies that the approximation which leads to the effective Dirac equation is well satisfied. (ii) We have also calculated the transmission coefficient for the weak atomic interaction in BECs in Fig. 14(b), which shows that the effect of the weak interaction is little and smooths merely the tunneling oscillation slightly. Therefore the exotic tunneling phenomena addressed here survive in the case of weak interaction between atoms.

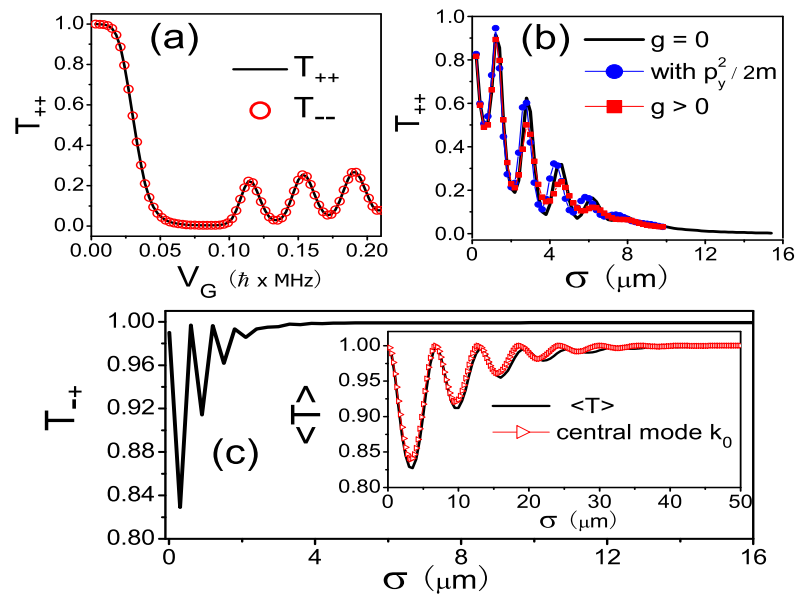

Fig. 14 Klein tunneling of BECs. (a) $T_{++}$as a function of the potential height $V_{G}$ with $\sigma=5 \mu \mathrm{m}$. The relation $T_{++}=T_{--}$ is confirmed. (b) $T_{++}$as a function of potential width $\sigma$ with $V_{\mathrm{G}}=0.2 \mathrm{MHz}$. The tunnelings of a BEC with the classic kinetic energy term and the weak atomic interaction $\left(N=2 \times 10^{4}, l_{\perp}=1.4\right.$ $\mu \mathrm{m}$ and $a_{s}=5 a_{0}$ with $a_{0}$ being the Bohr radius) are also depicted. (c) $T_{-+}$as a function of potential width $\sigma$ with $V_{\mathrm{G}}=0.2 \mathrm{MHz}$. In the insert, the transmission coefficient of a plane wave at bf the central mode $k_{0}$ is shown as the line with labels of triangle, while the average transmission coefficient $\langle T\rangle$ of $10^{4}$ atoms is shown as the solid line. The other parameters: $l_{0}=10 \mu \mathrm{m}, k_{0}=5.5 \times 10^{5}$ $\mathrm{m}^{-1}, \gamma_{z} / \hbar=30 \mathrm{kHz}$, and $d=4\left(l_{0}+\sigma\right)$.

\section{CONCLUSIONS AND PERSPECTIVES}

In summary, we have reviewed recent progress on quantum simulation of the relativistic Dirac equation by using ultracold neutral atoms. As we have shown, ultracold atoms in a wide range of systems, including the optical lattices with designated structures and different light-induced gauge fields, can behave as the relativistic particles under certain conditions. Comparing with other relativistic systems such as the graphene, cold atom systems offer rather more degrees of freedom to the relativistic quasiparticles, which can exhibit from one-dimension to three-dimension, and even from massive to massless. In other words, one can simulate a highly tunable Dirac equation with cold atoms by well dressing laser beams. Furthermore, the controllable atomic interactions and disorders in these systems provide a unique platform to investigate the relativistic dynamics and scattering processes such as the celebrated Zitterbewegung effect and Klein tunneling as well as its extended macroscopic version.

Remarkably, some crucial experimental techniques for cold atoms used to be Dirac equation simulators have been recently demonstrated. The important progress includes addressing atomic gases in a hexagonal optical lattice [69], and generating an effective U(2) non-Abelian gauge potential in a BEC [116]. The latter technique is principally available for fermionic atoms, which may provide an opportunity for the realization of the long-sought Majorana fermions (see Refs. $[145,146]$ and the referred works therein) in ultracold atomic superfluid [147-149]. The Majorana fermions described by the Majorana equation are distinguished from the Dirac fermions by the most exotic property that Majorana fermions are their own antiparticles. The Majorana fermions with the feature of non-Abelian statistics has raised significant interest as candidates for the realization of topological quantum computation [150]. To achieve this goal, we will still face the arduous task of manipulating Majorana fermions in cold atomic superfluid as that in other proposed systems [151]. Another important issue beyond the hunt for Majonana fermions should be the quantum simulation of Majoranna equation [152], which is central to the recent work not only in particle physics, supersymmetry and dark matter, but also some exotic states of ordinary matter. If succeed, we are allowed to explore some new exotic quantum relativistic processes that may go beyond ordinary Dirac quantum mechanics.

In the present proposals of quantum simulation of the Dirac equation, the atomic interactions are less considered due to the complexity such as the nonlinear interacting for bosonic atoms. Thus, it is of interest to ask whether and how the whole physics picture of relativistic dynamics in the present of controllable nonlinearity, that is an open question in the original Dirac theory, can be learned by cold atom simulators [105,120]. Besides, combining with the controllable laser-formed disorder potential techniques, we may also be able to investigate the 
Anderson localization of relativistic particles [103], especially the relativistic version of competition between the interaction and disorder, which is still a controversial problem even for the non-relativistic particles. Furthermore, shall we be able to realize the quantum simulation of interacting relativistic quantum field theories [153] or find some other exotic high-energy phenomena in these oppositely lowest-temperature systems among the universe?
Acknowledgements: This work was supported by the National Natural Science Foundation of China (Grant Nos. 10974059 and 11125417), the State Key Program for Basic Research of China (Grant No. 2011CB922104), the GRF and CRF of the RGC of Hong Kong.
[1] R. Feynman, Int. J. Theor. Phys., 1982, 21: 467

[2] See the reviews in Insight: Quantum Coherence, Nature, 2008, 453: 1003-1049

[3] I. Buluta and F. Nori, Science, 2009, 326: 108

[4] M. Greiner, O. Mandel, T. Esslinger, T. W. Hänsch, I. Bloch, Nature, 2002, 415: 39

[5] T. Stöferle, H. Moritz, C. Schori, M. Köhl, and T. Esslinger, Phys. Rev. Lett., 2004, 92: 130403

[6] I. B. Spielman, W. D. Phillips, and J.V. Porto, Phys. Rev. Lett., 2007, 98: 080404

[7] J. Simon, W. S. Bakr, R. Ma, M. E. Tai, P. M. Preiss, and M. Greiner, Nature, 2011, 472: 307

[8] A. Friedenauer, H. Schmitz, J. T. Glueckert, D. Porras, and T. Schaetz, Nature Phys., 2008, 4: 757

[9] K. Kim, M. S. Chang, S. Korenblit, R. Islam, E. E. Edwards, J. K. Freericks, G. D. Lin, L. M. Duan, and C. Monroe, Nature, 2010, 465: 590

[10] R. Gerritsma, G. Kirchmair, F. Zähringer, E. Solano, R. Blatt, and C. F. Roos, Nature, 2010, 463: 68

[11] R. Gerritsma, R. Gerritsma, B. P. Lanyon, G. Kirchmair, F. Zähringer, C. Hempel, J. Casanova, J. J. García-Ripoll, E. Solano, R. Blatt, and C. F. Roos, Phys. Rev. Lett., 2011, 106: 060503

[12] J. T. Barreiro, M. Müller, P. Schindler, D. Nigg, T. Monz, M. Chwalla, M. Hennrich, C. F. Roos, P. Zoller, and R. Blatt, Nature, 2011, 470: 486

[13] J. Du, N. Xu, X. Peng, P. Wang, S. Wu, and D. Lu, Phys. Rev. Lett., 2010, 104: 030502

[14] M. H. Anderson, J. R. Ensher, M. R. Matthews, C. E. Wieman, and E. A. Cornell, Science, 1995, 269: 198

[15] F. Dalfovo, S. Giorgini, L. P. Pitaevskii, and S. Stringari, Rev. Mod. Phys., 1999, 71: 463

[16] C. A. Regal, M. Greiner, and D. S. Jin, Phys. Rev. Lett., 2004, 92: 040403

[17] S. Giorgini, L. P. Pitaevskii, and S. Stringari, Rev. Mod. Phys., 2008, 80: 1215

[18] J. Liu and B Liu, Front. Phys. China, 2010, 5: 123

[19] H. Jing, Y. Jiang, and Y. Deng, Front. Phys., 2011, 6: 15

[20] M. Lewenstein, A. Sanpera, V. Ahufinger, B. Damski, A. Sende, U. Sen, Adv. Phys., 2007, 56: 243

[21] H. Zhai, Front. Phys. China, 2009, 4: 1

[22] A. Klein and D. Jaksch, Phys. Rev. A, 2006, 73: 053613

[23] M. A. Baranov, K. Osterloh, and M. Lewenstein, Phys. Rev. Lett., 2005, 94: 070404

[24] A. S. Sørensen, E. Demler, and M. D. Lukin, Phys. Rev. Lett., 2005, 94: 086803

[25] G. B. Jo, Y. R. Lee, J. H. Choi, C. A. Christensen, T. H. Kim, J. H. Thywissen, D. E. Pritchard, and W. Ketterle, Science, 2009, 325: 1521
[26] J. Billy, V. Josse, Z. Zuo, A. Bernard, B. Hambrecht, P. Lugan, D. Clément, L. Sanchez-Palencia, P. Bouyer, and A. Aspect, Nature, 2008, 453: 891

[27] G. Roati, C. DErrico, L. Fallani, M. Fattori, C. Fort, M. Zaccanti, G. Modugno, M. Modugno, and M. Inguscio, Nature, 2008, 453: 895

[28] B. Deissler, M. Zaccanti, G. Roati, C. D'Errico, M. Fattori, M. Modugno, G. Modugno, and M. Inguscio, Nature Phys., 2010, 6: 354

[29] L. Sanchez-Palencia and M Lewenstein, Nature Phys., 2010, 6: 87

[30] L. J. Garay, J. R. Anglin, J. I. Cirac, and P. Zoller, Phys. Rev. Lett., 2000, 85: 4643

[31] U. R. Fischer and R. Schützhold, Phys. Rev. A, 2004, 70: 063615

[32] M. Snoek, M.Haque, S. Vandoren, and H. T. C. Stoof, Phys. Rev. Lett., 2005, 95: 250401

[33] Y. Yu and K. Yang, Phys. Rev. Lett., 2008, 100: 090404

[34] Y. Yu and K. Yang, Phys. Rev. Lett., 2010, 105: 150605

[35] K. S. Novoselov, A. K. Geim, S. V. Morozov, D. Jiang, Y. Zhang, S. V. Dubonos, I. V. Grigorieva, and A. A. Firsov, Science, 2004, 306: 666

[36] K. S. Novoselov, A. K. Geim, S. V. Morozov, D. Jiang, M. I. Katsnelson, I. V. Grigorieva, S. V. Dubonos, and A. A. Firsov, Nature, 2005, 438: 197

[37] M. Z. Hasan, C. L. Kane, Rev. Mod. Phys., 2010, 82: 3045

[38] W. Greiner, Relativistic Quantum Mechanics, 3rd Ed., Berlin: Spinger-Verleg, 2003

[39] E. Schrödinger, Sitzungsber. Preuss. Akad. Wiss. Phys. Math. Kl., 1930, 24: 418

[40] O. Klein, Z. Phys., 1929, 50: 157

[41] S. L. Zhu, B. Wang, and L. M. Duan, Phys. Rev. Lett., 2007, 98: 260402

[42] E. Zhao and A. Paramekanti, Phys. Rev. Lett., 2006, 97: 230404

[43] C. Wu and S. Das Sarma, Phys. Rev. B, 2008, 77: 235107

[44] B. Wunsch, F. Guinea, and F. Sols, New J. Phys., 2008, 10: 103027

[45] K. L. Lee, B. Grémaud, R. Han, B. G. Englert, and C. Miniatura, Phys. Rev. A, 2009, 80: 043411

[46] A. Dutta, R. R. P. Singh, and U. Divakaran, Europhys. Lett., 2010, 89: 67001

[47] D. Poletti, C. Miniatura, and B. Grémaud, Europhys. Lett., 2011, 93: 37008

[48] D. Bercioux, D. F. Urban, H. Grabert, and W. Häsler, Phys. Rev. A, 2009, 80: 063603

[49] D. Bercioux, N. Goldman, and D. F. Urban, Phys. Rev. A, 2011, 83: 023609 
[50] R. Shen, L. B. Shao, B. Wang, and D. Y. Xing, Phys. Rev. B, 2010, 81: 041410(R)

[51] I. I. Satija, D. C. Dakin, J. Y. Vaishnav, and C. W. Clark, Phys. Rev. A, 2008, 77: 043410

[52] J. M. Hou, W. X. Yang, and X. J. Liu, Phys. Rev. A, 2009, 79: 043621

[53] L. K. Lim, C. M. Smith, and A. Hemmerich, Phys. Rev. Lett., 2008, 100: 130402

[54] L. K. Lim, A. Hemmerich, and C. M. Smith, Phys. Rev. A, 2010, 81: 023404

[55] L. K. Lim, A. Lazarides, A. Hemmerich, and C. M. Smith, Europhys. Lett., 2009, 88: 36001

[56] N. Goldman, A. Kubasiak, A. Bermudez, P. Gaspard, M. Lewenstein, and M. A. Martin-Delgado, Phys. Rev. Lett., 2009, 103: 035301

[57] X. J. Liu, X. Liu, C. Wu, and J. Sinova, Phys. Rev. A, 2010, 81: 033622

[58] M. P. Kennett, N. Komeilizadeh, K. Kaveh, and P. M. Smith, Phys. Rev. A, 2011, 83: 053636

[59] M. Yang and S. L. Zhu, Phys. Rev. A, 2010, 82: 064102

[60] A. Bermudez, L. Mazza, M. Rizzi, N. Goldman, M. Lewenstein, and M. A. Martin-Delgado, Phys. Rev. Lett., 2010, 105: 190404

[61] L. Lepori, G. Mussardo, and A. Trombettoni, Europhys. Lett., 2010, 92: 50003

[62] L. M. Duan, E. Demler, and M. D. Lukin, Phys. Rev. Lett., 2003, 91: 090402

[63] Y. Zhang, Y. Tan, H. L. Stormer, and P. Kim, Nature, 2005, 438: 201

[64] G. W. Semenoff, Phys. Rev. Lett., 1984, 53: 2449

[65] X. G. Wen, Quantum Field Theory of Many-Body Systems, Oxford: Oxford University, 2004.

[66] A. Bermudez, N. Goldman, A. Kubasiak, M. Lewenstein, and M. A. Martin-Delgado, New J. Phys., 2010, 12: 033041

[67] J. K. Block and N. Nygaard, Phys. Rev. A, 2010, 81: 053421

[68] C. Wu, D. Bergman, L. Balents, and S. Das Sarma, Phys. Rev. Lett., 2007, 99: 070401

[69] P. Soltan-Panahi, J. Struck, P. Hauke, A. Bick, W. Plenkers, G. Meineke, C. Becker, P. Windpassinger, M. Lewenstein and K. Sengstock , Nature Phys., 2011, 7: 434

[70] V. Apaja, M. Hyrkäs, and M. Manninen, Phys. Rev. A, 2010, 82: 041402(R)

[71] D. Jaksch and P. Zoller, New J. Phys., 2003, 5: 56

[72] K. Osterloh, M. Baig, L. Santos, P. Zoller, and M. Lewenstein, Phys. Rev. Lett., 2005, 95: 010403

[73] F. Gerbier and J. Dalibard, New J. Phys., 2010, 12: 033007

[74] N. Goldman, A. Kubasiak, P. Gaspard, and M. Lewenstein, Phys. Rev. A, 2009, 79: 023624

[75] L. H. Karsten and J. Smith, Nucl. Phys. B, 1981, 183 : 103

[76] H. B. Nielsen and M. Ninomiya, Nucl. Phys. B, 1981, 185: 20

[77] K. Wilson, New Phenomena in Subnuclear Physics., edited Plenum: New York, 1977

[78] X. L. Qi, R. Li, J. Zang, S. C. Zhang, Science, 2009, 323: 1184

[79] M. W. Zwierlein, A. Schirotzek, C. H. Schunck, and W. Ketterle, Science, 2006, 311: 492

[80] J. Stenger, S. Inouye, A. P. Chikkatur, D. M. StamperKurn, D. E. Pritchard, and W. Ketterle, Phys. Rev.
Lett., 1999, 82: 4569

[81] F. D. M. Haldane, Phys. Rev. Lett., 1988, 61: 2015

[82] M. Onoda and N. Nagaosa, Phys. Rev. Lett., 2003, 90: 206601

[83] C. X. Liu, X. L. Qi, X. Dai, Z. Fang, and S. C. Zhang, Phys. Rev. Lett., 2008, 101: 146802

[84] R. Yu, W. Zhang, H. J. Zhang, S. C. Zhang, X. Dai, and Z. Fang, Science, 2010, 329: 61

[85] Z. Qiao, S. A. Yang, W. Feng, W. K. Tse, J. Ding, Y. Yao, J. Wang, and Q. Niu, Phys. Rev. B, 2010, 82: 161414

[86] Y. Zhang and C. Zhang, Phys. Rev. B, 2011, 84: 085123

[87] H. Li, L. Sheng, and D. Y. Xing, Phys. Rev. B, 2011, 84: 035310

[88] L. B. Shao, S. L. Zhu, L. Sheng, D. Y. Xing, and Z. D. Wang, Phys. Rev. Lett., 2008, 101: 246810

[89] C. Wu, Phys. Rev. Lett., 2008, 101: 186807

[90] N. Gemelke, Ph.D. thesis, Stanford University, 2007

[91] M. Zhang, Hsiang-hsuan Hung, C. Zhang, and C. Wu, Phys. Rev. A, 2011, 83: 023615

[92] X. J. Liu, X. Liu, C. Wu, and J. Sinova, Phys. Rev. A, 2010, 81: 033622

[93] G. Juzeliūnas and P. Öhberg, Phys. Rev. Lett., 2004, 93: 033602

[94] G. Juzeliūnas, P. Öhberg, J. Ruseckas, and A. Klein, Phys. Rev. A, 2005, 71: 053614

[95] P. Öhberg, G. Juzeliūnas, J. Ruseckas, and M. Fleischhauer, Phys. Rev. A, 2005, 72: 053632

[96] G. Juzeliūnas, J. Ruseckas, P. Öhberg, and M. Fleischhauer, Phys. Rev. A, 2006, 73: 025602

[97] S. L. Zhu, H. Fu, C. J. Wu, S. C. Zhang, and L. M. Duan, Phys. Rev. Lett., 2006, 97: 240401

[98] K. J. Günter, M. Cheneau, T. Yefsah, S. P. Rath, and J. Dalibard, Phys. Rev. A, 2009, 79: 011604(R)

[99] J. Dalibard, F. Gerbier, G. Juliūnas, and P. Öhberg, arXiv: cond-mat/1008.5378

[100] J. Ruseckas, G. Juzeliūnas, P. Öhberg, and M. Fleischhauer, Phys. Rev. Lett., 2005, 95: 010404

[101] F. Wilczek and A. Zee, Phys. Rev. Lett., 1984, 52: 2111

[102] G. Juzeliūnas, J. Ruseckas, M. Lindberg, L. Santos, and P. Öhberg, Phys. Rev. A, 2008, 77: 011802(R)

[103] S. L. Zhu, D. W. Zhang, and Z. D. Wang, Phys. Rev. Lett., 2009, 102: 210403

[104] M. Merkl, F. E. Zimmer, and P. Öhberg, Europhys. Lett., 2008, 83: 54002

[105] D. W. Zhang, Z. Y. Xue, H. Yan, Z. D. Wang, and S. L. Zhu, arXiv: cond-mat/1104.0444

[106] N. R. Cooper, Adv. Phys., 2008, 57: 539

[107] M. Burrello and A. Trombettoni, Phys. Rev. Lett., 2010, 105: 125304

[108] X. J. Liu, X. Liu, L. C. Kwek, C. H. Oh, Front. Phys., 2008, 3(2): 113

[109] I. B. Spielman, Phys. Rev. A, 2009, 79: 063613

[110] Y. J. Lin, R. L. Compton, A. R. Perry, W. D. Phillips, J. V. Porto, and I. B. Spielman, Phys. Rev. Lett., 2009, 102: 130401

[111] Y. J. Lin, R. L. Compton, K. Jiménez-García, J. V. Porto, and I. B. Spielman, Nature, 2009, 462: 628

[112] Y. J. Lin, R. L. Compton, K. Jiménez-García, W. D. Phillips, J. V. Porto, and I. B. Spielman, Nature Phys., 2011, 7: 531

[113] T. D. Stanescu, B. Anderson, and V. Galitski, Phys. Rev. A, 2008, 78: 023616 
[114] C. J. Wang, C. Gao, C. M. Jian and H. Zhai, Phys. Rev. Lett., 2010, 105: 160403

[115] T. L. Ho and S. Zhang, arXiv: cond-mat/1007.0650

[116] Y. J. Lin, K. Jiménez-García, and I. B. Spielman, Nature, 2011, 471: 83

[117] X. J. Liu, M. F. Borunda, X. Liu and J. Sinova, Phys. Rev. Lett., 2009, 102: 046402

[118] G. Juzeliūnas, J. Ruseckas, and J. Dalibard, Phys. Rev. A, 2010, 81: 053403

[119] Z. Lan, N. Goldman, A. Bermudez, W. Lu, and P. Öhberg, Phys. Rev. B, 2011, 84: 165115

[120] M. Merkl, A. Jacob, F. E. Zimmer, P. Ö hberg, and L. Santos, Phys. Rev. Lett., 2010, 104: 073603

[121] L. H. Haddad and L. D. Carr, Physica D: Nonlinear Phenomena, 2009, 238: 1413

[122] L. H. Haddad and L. D. Carr, Europhys. Lett., 2011, 94: 56002

[123] Z. Chen and B. Wu, Phys. Rev. Lett., 2011, 107: 065301

[124] See the review, W. Zawadzki and T. M Rusin, J. Phys.: Condens. Matter, 2011, 23: 143201

[125] J. Schliemann, D. Loss, and R. M. Westervelt, Phys. Rev. Lett., 2005, 94: 206801

[126] M. I. Katsnelson, Eur. Phys. J. B, 2006, 51: 157

[127] B. Trauzettel, Y. M. Blanter, and A. F. Morpurgo, Phys. Rev. B, 2007, 75: 035305

[128] D. Lurié and S. Cremer, Physica, 1970, 50: 224

[129] X. Zhang, Phys. Rev. Lett., 2008, 100: 113903

[130] F. Dreisow, M. Heinrich, R. Keil, A. Tünnermann, S. Nolte, S. Longhi, and A. Szameit, Phys. Rev. Lett., 2010, 105: 143902

[131] L. Lamata, J. León, T. ScHätz, and E. Solano, Phys. Rev. Lett., 2007, 98: 253005

[132] J. Y. Vaishnav and C. W. Clark, Phys. Rev. Lett., 2008, 100: 153002

[133] J. J. Song and B. A. Foreman, Phys. Rev. A, 2009, 80: 045602

[134] Q. Zhang, J. Gong, and C. H. Oh, Phys. Rev. A, 2010, 81,023608

[135] D. Witthaut, Phys. Rev. A, 2010, 82: 033602

[136] J. Larson, J. P. Martikainen, A. Collin and E. Sjöqvist, Phys. Rev. A, 2010, 82: 043620
[137] M. I. Katsnelson, K. S. Novoselov, and A. K. Geim, Nature Phys., 2006, 2: 620

[138] O. Bahat-Treidel, O. Peleg, M. Grobman, N. Shapira, M. Segev, and T. Pereg-Barnea, Phys. Rev. Lett., 2010, 104: 063901

[139] D. Witthaut, T. Salger, S. Kling, C. Grossert, and M. Weitz, Phys. Rev. A, 2011, 84: 033601

[140] T. Salger, C. Grossert, S. Kling, and M. Weitz, arXiv: cond-mat/1108.4447

[141] J. Otterbach, R. G. Unanyan, and M. Fleischhauer, Phys. Rev. Lett., 2009, 102: 063602

[142] M. G. Tarallo, J. Miller, J. Agresti, E. D'Ambrosio, R. DeSalvo, D. Forest, B. Lagrange, J. M. Mackowsky, C. Michel, J. L. Montorio , N. Morgado, L. Pinard, A. Remilleux, B. Simoni, P. Willems, Appl. Opt., 2007, 46: 3348

[143] L. Khaykovich, F. Schreck, G. Ferrari, T. Bourdel, J. Cubizolles, L. D. Carr, Y. Castin, and C. Salomon, Science, 2002, 296: 1290

[144] N. Dombey, P. Kennedy, and A. Calogeracos, Phys. Rev. Lett., 2000, 85: 1787

[145] F. Wilczek, Nature Phys., 2009, 5: 614

[146] R. F. Service, Science, 2011, 332: 193

[147] S. L. Zhu, L. B. Shao, Z. D. Wang, and L. M. Duan, Phys. Rev. Lett., 2011, 106: 100404

[148] L. Jiang, T. Kitagawa, J. Alicea, A. R. Akhmerov, D. Pekker, G. Refael, J. I. Cirac, E. Demler, M. D. Lukin, and P. Zoller, Phys. Rev. Lett., 2011, 106: 220402

[149] S. Tewari, S. Das. Sarma, C. Nayak, C. Zhang, and P. Zoller, Phys. Rev. Lett., 2007, 98: 010506

[150] C. Nayak, S. H. Simon, A. Stern, M. Freedman, and S. Das. Sarma, Rev. Mod. Phys., 2008, 80: 1083

[151] J. Alicea, Y. Oreg, G. Refael, F. von Oppen and M. P. A. Fisher, Nature Phys., 2011, 7: 412

[152] J. Casanova, C. Sabin, J. Leon, I. L. Egusquiza, R. Gerritsma, C. F. Roos, J. J. Garcia-Ripoll, E. Solano, arXiv: 1102.1651, 2011

[153] J. I. Cirac, P. Maraner, and J. K. Pachos, Phys. Rev. Lett., 2010, 105: 190403 